\title{
Quantum trajectories: memory and continuous observation
}

\author{
Alberto Barchielli \\ Politecnico di Milano, Dipartimento di Matematica, \\ Piazza Leonardo da Vinci 32, I-20133 Milano, Italy \\ Clément Pellegrini \\ Laboratoire de Statistique et Probabilités, Université Paul Sabatier, \\ 118, Route de Narbonne, 31062 Toulouse Cedex 4, France. \\ Francesco Petruccione \\ University of KwaZulu-Natal, School of Physics and National Institute for Theoretical Physics, \\ Private Bag X54001, Durban 4000, South Africa.
}

(Dated: January 22, 2018)

\begin{abstract}
Starting from a generalization of the quantum trajectory theory [based on the stochastic Schrödinger equation (SSE)], non-Markovian models of quantum dynamics are derived. In order to describe non-Markovian effects, the approach used in this article is based on the introduction of random coefficients in the usual linear SSE. A major interest is that this allows a consistent theory of quantum measurement in continuous time to be developed for these non-Markovian quantum trajectory models. In this context, the notions of 'instrument', 'a priori', and 'a posteriori' states can be introduced. The key point is that by starting from a stochastic equation on the Hilbert space of the system, we are able to respect the complete positivity of the mean dynamics for the statistical operator and the requirements of the axioms of quantum measurement theory. The flexibility of the theory is next illustrated by a concrete physical model of a noisy oscillator where non-Markovian effects come from the random environment, colored noises, randomness in the stimulating light, and delay effects. The statistics of the emitted photons and the heterodyne and homodyne spectra are studied, and we show how these quantities are sensitive to the non-Markovian features of the system dynamics, so that, in principle, the observation and analysis of the fluorescent light could reveal the presence of non-Markovian effects and allow for a measure of the spectra of the noises affecting the system dynamics.
\end{abstract}

PACS numbers: 42.50.Lc, 03.65.Ta, 03.65.Yz

\section{INTRODUCTION}

A first aim of the theory of open quantum systems is the description of the time evolution of a quantum system $\mathcal{S}$ (the open system) interacting with an environment $\mathcal{E}$ [1]. More precisely, one focuses on the reduced evolution of $\mathcal{S}$ after tracing out the degrees of freedom of $\mathcal{E}$. The resulting evolution is then usually described in terms of generalized master equations for the reduced density matrix $\rho(t)$.

A particular simple and useful way to describe an open system is provided by the Markovian approximation [2]. Essentially, this approach is based on the absence of memory effects in the environment. In this situation, the master equations are linear first-order differential equations with a possibly time dependent generator. The generator takes a particular form, the well known Lindblad form, that guarantees the complete positivity of the dynamics, as required by quantum mechanics.

Unfortunately, this approximation is no longer valid when the memory effects of the environment cannot

\footnotetext{
* Also at Istituto Nazionale di Fisica Nucleare (INFN), Sezione di Milano, and Istituto Nazionale di Alta Matematica (INDAMGNAMPA).
}

be neglected. Several physical situations involve longmemory-time effects and lead to a non-Markovian behavior: strong coupling, correlation, and entanglement in the initial $\mathcal{S}-\mathcal{E}$ state [3], a? system at low temperature [4], and a? structured environment [5]. In this context, the master equations take different forms according to the physical situation, e.g., integro-differential equations [1, 6], time-convolutionless equations [1], and Lindblad rate equations [7]. The common point is that they are not in the Lindblad form, which is characteristic of Markovian evolutions.

In both situations (Markovian and non-Markovian) a useful approach to describe concrete physical evolutions is provided by the theory of the stochastic Schrödinger equation (SSE) 8-11]. A SSE is a nonlinear stochastic differential equation for a wave-function process $\psi(t)$. The link with the traditional master equation is given by the average property $\mathbb{E}[|\psi(t)\rangle\langle\psi(t)|]=\rho(t)$, where $\mathbb{E}$ denotes the average over the realizations of $\psi(t)$. To find the SSE providing a given master equation by averaging is called unraveling. The idea of unraveling has been a real breakthrough for simulating master equations; it is at the root of the Monte-Carlo wave function method [1, 12]. Indeed, for huge systems, the description of $\psi(t)$ requires many fewer parameters than the ones needed for $\rho(t)$. 
However, the construction of adequate SSEs has been essential also for a second aspect of the theory of open quantum systems: the description of the monitoring of $\mathcal{S}$. In special situations, the SSE can be interpreted in terms of quantum measurements. More precisely, in these cases, the solution $\psi(t)$ is called a quantum trajectory and describes the evolution of an open system undergoing indirect continuous measurement (continuous monitoring) [10, 13 15]. In particular the noises involved in the SSE, describing jump or diffusion evolutions, can be directly connected with the outputs of measurement apparatuses. Such an interpretation is crucial in the understanding of real quantum optics experiments [10, 11, 14, 16, 17] such as direct photo-detection, spectral photo-detection, homodyning, and heterodyning. They are also at the cornerstone of modern technology such as feedback control [8, 15, 18]. As a consequence an active line of research consists in finding SSEs that can be physically interpreted in terms of continuous monitoring of the system.

In the Markovian case, this link is clearly established for almost all situations 13 15. Starting from a master equation in Lindblad form, it is known how to construct an appropriate unraveling in terms of a SSE. The (nonlinear) SSE is a stochastic equation for a random normalized vector $\psi(t)$. It is always possible to construct a linear SSE, driven by Poisson and Wiener noises, for a nonnormalized vector $\phi(t)$, such that $\psi(t)=\phi(t) /\|\phi(t)\|$. Moreover, the linear and nonlinear versions of the SSE are related by a change of probability measure and it is this link that allows for a measurement interpretation consistent with the postulates of quantum mechanics [10, 14]. In mathematical terms the change of measure is a Girsanov transformation with probability density $\|\phi(t)\|^{2}$; the key point that allows this transformation is the fact that $\|\phi(t)\|^{2}$ turns out to be a martingale 14 . Moreover, these stochastic differential equations can be deduced from purely quantum evolution equations for the measured system coupled with a quantum environment, combined with a continuous monitoring of the environment itself [19, 20].

In the non-Markovian case, to find relevant SSEs, describing both non-Markovian quantum evolutions and continuous monitoring, is a tremendous challenge. In contrast to the Markovian framework, no general theory has been developed. Essentially there exist two strategies.

The first strategy consists in considering a physical model described by a non-Markovian master equation and in finding an appropriate pure-state unraveling. This approach has been successfully applied in various situations. A major common point consists in replacing the memoryless white noises, used in Markovian SSEs, by colored Gaussian noises and in introducing some delay effects [21]. This allows the introduction of correlations in time that describe strong memory effects of the environment interaction. In this direction several models, such as, for example, the so-called non-Markovian quantum state diffusion, have been derived [22]. Other investigations involving non-Markovian jump type SSEs have been also proposed [23, 24]. While such approaches are efficient for simulating relevant non-Markovian evolutions, the measurement interpretation of the underlying SSEs is highly debated [24 26] and a complete conclusion is still lacking. A principal problem concerns the interpretation of the underlying noises as outputs of continuoustime measurements. For other models such as Lindblad rate equations, different types of jump unravelings have been proposed [27]. Jump-diffusion generalizations with measurement applications have been derived in Ref. [28]. In this context limitations also appear in the sense that the types of observables that can be measured must have particular and restrictive forms.

A second strategy is first to generalize directly the Markovian SSE by introducing memory effects. Then, one has to show whether this SSE provides the unraveling of some non-Markovian evolution and whether it has a physical measurement interpretation. To work at the Hilbert space level guarantees automatically the complete positivity of the evolution of the statistical operator. In this paper we propose non-Markovian SSE models with physical measurement interpretations. Our strategy consists in adapting the Markovian approach by replacing white and Poisson noises with non-Markovian noises and by allowing for random coefficients in the equation. First we start with a linear SSE driven by colored noises and involving random operator coefficients; the whole randomness is defined under a reference probability. Next we introduce the physical probability and the nonlinear SSE, which is a stochastic differential equation under the new probability. Finally it is possible to pass to the linear and nonlinear versions of the stochastic master equation (SME), and we show that they determine the dynamics and the continuous measurements without violating the axiomatic structure of quantum mechanics. The general mathematical structure was introduced in 29, 30]; in 31] we started to show how such a structure allows for the introduction of some colored noises, while in [32] we considered memory effects due to feedback with delay. The present article is devoted to the exploration and clarification of physical effects that can be treated within such a theory and to show them on a concrete physical system.

The paper is structured as follows. Section $\Pi$ describes the general theory of the stochastic Schrödinger equation. We present the general mathematical ingredients necessary to develop the generalization of the SSE involving colored noises and random coefficients. We consider first a linear stochastic equation for a non normalized wave function $\phi(t)$. Then, with the help of a change of measure, determined by the SSE itself, we derive the nonlinear SSE for the wave function $\psi(t)=\phi(t) /\|\phi(t)\|$. In Sec. III the linear SME and the nonlinear one are introduced and the measurement interpretation is justified by introducing positive operator-valued measures, instruments, a priori states (mean states), and a posteriori states (conditional states). Section IV is devoted to a 
concrete model, a noisy oscillator absorbing and emitting light, by which physical effects can be discussed and the possibilities of the theory can be explored. Moreover, in this section we study the behavior of the outputs of the oscillator; in particular we study the effects of the nonMarkovian terms in the dynamics on the homodyne and the heterodyne spectra of the emitted light and on the statistics of the photons, now analyzed by direct detection. Conclusions are presented in Sec. V.

\section{THE STOCHASTIC SCHRÖDINGER EQUATION}

When introducing non-Markovian evolutions for a quantum state, the first problem is to guarantee the complete positivity of the evolution of the state (statistical operator) of the reduced system. Then, if one wants to introduce measurements in continuous time, the second problem is to have equations compatible with quantum measurement theory. Starting from the linear version of the SSE allows memory to be introduced by using random coefficients and colored noises (no problem of complete positivity because we are working at Hilbert space level) and the instruments related to the continuous monitoring to be constructed (no problem with the axioms of quantum theory because we are respecting linearity) 29 31].

Let us denote by $\mathscr{H}$ the Hilbert space of the quantum system of interest, a separable complex Hilbert space, by $\mathscr{L}(\mathscr{H})$ the space of the bounded operators on $\mathscr{H}$, by $\mathscr{T}(\mathscr{H}) \subset \mathscr{L}(\mathscr{H})$ the trace class and by $\mathscr{S}(\mathscr{H}) \subset$ $\mathscr{T}(\mathscr{H})$ the convex set of the statistical operators.

\section{A. The linear SSE and the reference probability}

The starting point of the whole construction is the linear SSE for a stochastic process $\phi(t)$ with values in $\mathscr{H}$ :

$$
\begin{aligned}
\mathrm{d} \phi(t)=K_{0}(t) \phi\left(t_{-}\right) & \mathrm{d} t+\sum_{j=1}^{m} L_{j}^{0}(t) \phi\left(t_{-}\right) \mathrm{d} M_{j}(t) \\
& +\sum_{k=1}^{d^{\prime}}\left(R_{k}(t)-\mathbb{1}\right) \phi\left(t_{-}\right) \mathrm{d} N_{k}(t) .
\end{aligned}
$$

In the Markovian case the $M_{j}$ are Wiener processes, the $N_{k}$ are Poisson processes, and all these processes are independent. Moreover, the operators $R_{k}(t), L_{j}^{0}(t)$, and $K(t)$ are not random. The key property that allows for a measurement interpretation is that $\|\phi(t)\|^{2}$ is a mean-1 martingale, and this requirement imposes a link among the operators $R_{k}(t), L_{j}^{0}(t)$, and $K_{0}(t)$. The nonMarkovian generalization is to take more general processes as driving noises and to allow for random coefficients. Now we illustrate the precise meaning of the various quantities appearing in the SSE (1).
First of all we work in a reference probability space $(\Omega, \mathscr{F}, \mathbb{Q}) ; \Omega$ is the sample space, $\mathscr{F}$ the $\sigma$-algebra of events, and $\mathbb{Q}$ a reference probability. The physical probability will appear when the measuring interpretation is constructed in Sec.IIB Past and present up to time $t$ are represented by the events in the sub- $\sigma$-algebra $\mathscr{F}_{t} \subset \mathscr{F}$; the family $\left(\mathscr{F}_{t}\right)_{t \geq 0}$ is a filtration of $\sigma$-algebras satisfying the usual hypotheses, i.e., $\mathscr{F}_{s} \subset \mathscr{F}_{t}$ for $0 \leq s<t, A \in \mathscr{F}$ with $\mathbb{Q}(A)=0$ implies $A \in \mathscr{F}_{0}$, and $\mathscr{F}_{t}=\bigcap_{T>t} \mathscr{F}_{T}$. In $\left(\Omega, \mathscr{F},\left(\mathscr{F}_{t}\right)_{t}, \mathbb{Q}\right)$ we have $d$ continuous, independent, adapted, standard Wiener processes $B_{1}, \ldots, B_{d}$ and $d^{\prime}$ adapted càdlàg counting process of stochastic intensities $i_{k}(t) \geq 0$, that are càglàd. The French acronym càdlàg means with trajectories continuous from the right and with limits from the left, while càglàd means continuous from the left and with limits from the right. The meaning of stochastic intensity is given by the heuristic conditional expectation

$$
\mathbb{E}_{\mathbb{Q}}\left[\mathrm{d} N_{k}(t) \mid \mathscr{F}_{t}\right]=i_{k}(t) \mathrm{d} t
$$

the stochastic intensities determine the probability law of the counting processes [33]. Assuming the usual hypothesis, that the processes are càdlàg or càglàd, etc., are mathematical regularity requirements useful in a rigorous development of stochastic calculus, what is physically important is to have non anticipating (= adapted) processes.

The $m$ continuous processes $M_{j}(t)$ are given by

$$
M_{j}(t)=\int_{0}^{t} f_{j}(s) \mathrm{d} s+\sum_{i=1}^{d} \int_{0}^{t} b_{j i}(s) \mathrm{d} B_{i}(s),
$$

where $f_{j}(t)$ and $b_{j k}(t)$ are complex, adapted càglàd processes such that $\forall t>0$, with probability $1, \int_{0}^{t}\left|f_{j}(t)\right| \mathrm{d} t<$ $+\infty$ and $\int_{0}^{t}\left|b_{j i}(t)\right|^{2} \mathrm{~d} t<+\infty$. Some typical choices are given in Sec. IV.

The functions $t \mapsto L_{j}^{0}(t)$, and $t \mapsto R_{k}(t), t \mapsto K_{0}(t)$ are strongly càglàd, bounded operator-valued adapted processes; to be bounded is a sufficient condition to have a well-defined general equation [30]. For physical problems also the unbounded case is important and, indeed, the examples we shall give involve unbounded operators; the case involving unbounded operators, but restricted to a Markovian dynamics, is treated in [20, 34]. By allowing for random system operators and the general noises (3), it is possible to describe random external forces, random environments, colored baths, stochastic control, adaptive measurements and so on.

The SSE (11) is a linear stochastic differential equation in the Itô sense. The initial condition is taken to be

$$
\phi(0)=\phi_{0} \quad \text { with } \quad \mathbb{E}_{\mathbb{Q}}\left[\left\|\phi_{0}\right\|^{2}\right]=1 .
$$

Note that, by suitably choosing $\mathscr{F} 0, \mathbb{Q}$ and $\phi_{0}$, any statistical operator $\rho_{0} \in \mathscr{S}(\mathscr{H})$ can be represented as $\rho_{0}=\mathbb{E}_{\mathbb{Q}}\left[\left|\phi_{0}\right\rangle\left\langle\phi_{0}\right|\right]$. The solution $\phi(t)$ is taken to be càdlàg and it is unique [30]. To write $\phi\left(t_{-}\right)$means to take the 
value of $\phi$ just before the possible jump at time $t$ due to the counting processes.

By using the explicit expressions for the processes $M_{j}$, the linear SSE can be rewritten as

$$
\begin{aligned}
\mathrm{d} \phi(t)=K(t) \phi\left(t_{-}\right) & \mathrm{d} t+\sum_{i=1}^{d} L_{i}(t) \phi\left(t_{-}\right) \mathrm{d} B_{i}(t) \\
& +\sum_{k=1}^{d^{\prime}}\left[R_{k}(t)-\mathbb{1}\right] \phi\left(t_{-}\right) \mathrm{d} N_{k}(t),
\end{aligned}
$$

where $K(t)=K_{0}(t)+\sum_{j=1}^{m} f_{j}(t) L_{j}^{0}(t)$ and

$$
L_{i}(t)=\sum_{j=1}^{m} L_{j}^{0}(t) b_{j i}(t)
$$

For the physical interpretation in terms of measurements, we need $\|\phi(t)\|^{2}$ to be a martingale: $\mathbb{E}_{\mathbb{Q}}\left[\|\phi(t)\|^{2} \mid \mathscr{F}_{s}\right]=\|\phi(s)\|^{2}$, for $0 \leq s<t[14]$. We shall see that this is crucial for defining physical probabilities. To this end we have to compute $\mathrm{d}\|\phi(t)\|^{2}$. Here and in all the formulas involving stochastic differentials, we have to use Itô's formula and the rules of stochastic calculus, which are summarized by Itô's table

$$
\begin{gathered}
\mathrm{d} B_{i}(t) \mathrm{d} B_{j}(t)=\delta_{i j} \mathrm{~d} t, \quad \mathrm{~d} N_{k}(t) \mathrm{d} N_{j}(t)=\delta_{k j} \mathrm{~d} t \\
\mathrm{~d} B_{i}(t) \mathrm{d} N_{k}(t)=\mathrm{d} B_{i}(t) \mathrm{d} t=\mathrm{d} N_{k}(t) \mathrm{d} t=0 .
\end{gathered}
$$

Then, we get

$$
\begin{gathered}
\mathrm{d}\|\phi(t)\|^{2}=\sum_{i=1}^{d}\left\langle\phi\left(t_{-}\right) \mid\left[L_{i}(t)+L_{i}(t)^{\dagger}\right] \phi\left(t_{-}\right)\right\rangle \mathrm{d} B_{i}(t) \\
+\left\langle\phi\left(t_{-}\right) \mid\left(K(t)^{\dagger}+K(t)+\sum_{i=1}^{d} L_{i}(t)^{\dagger} L_{i}(t)\right) \phi\left(t_{-}\right)\right\rangle \mathrm{d} t \\
+\sum_{k=1}^{d^{\prime}}\left(\left\|R_{k}(t) \phi\left(t_{-}\right)\right\|^{2}-\left\|\phi\left(t_{-}\right)\right\|^{2}\right) \mathrm{d} N_{k}(t) .
\end{gathered}
$$

The martingale property is ensured if we have $\mathbb{E}_{\mathbb{Q}}\left[\mathrm{d}\|\phi(t)\|^{2} \mid \mathscr{F}_{t}\right]=0$. By (2) and $\mathbb{E}_{\mathbb{Q}}\left[\mathrm{d} B_{i}(t) \mid \mathscr{F}_{t}\right]=0$, we get the restriction

$$
\begin{aligned}
K(t)=-\mathrm{i} H(t)- & \frac{1}{2} \sum_{i=1}^{d} L_{i}(t)^{\dagger} L_{i}(t) \\
& +\frac{1}{2} \sum_{k=1}^{d^{\prime}} i_{k}(t)\left(\mathbb{1}-R_{k}(t)^{\dagger} R_{k}(t)\right)
\end{aligned}
$$

with $H(t)^{\dagger}=H(t)$.

\section{B. The nonlinear SSE and the physical probability}

Let us define the quantity

$$
p(t):=\|\phi(t)\|^{2}, \quad \forall t \geq 0,
$$

and the normalized version of $\phi(t)$,

$$
\psi(t, \omega):= \begin{cases}\|\phi(t, \omega)\|^{-1} \phi(t, \omega) & \text { if } p(t, \omega) \neq 0, \\ z & \text { if } p(t, \omega)=0,\end{cases}
$$

where $z \in \mathscr{H}$ is a non random vector with $\|z\|=1$ and we denote by $\omega$ the generic sample point in $\Omega$, as usual. Moreover, we introduce the processes

$$
\begin{gathered}
m_{i}(t):=2 \operatorname{Re}\left\langle\psi\left(t_{-}\right) \mid L_{i}(t) \psi\left(t_{-}\right)\right\rangle, \\
j_{k}(t):=i_{k}(t)\left\|R_{k}(t) \psi\left(t_{-}\right)\right\|^{2} .
\end{gathered}
$$

By condition (8), Eq. (7) becomes

$$
\begin{aligned}
& \mathrm{d} p(t)=p\left(t_{-}\right)\left\{\sum_{i=1}^{d} m_{i}(t) \mathrm{d} B_{i}(t)\right. \\
& \left.+\sum_{k=1}^{d^{\prime}}\left(\left\|R_{k}(t) \psi\left(t_{-}\right)\right\|^{2}-1\right)\left(\mathrm{d} N_{k}(t)-i_{k}(t) \mathrm{d} t\right)\right\} .
\end{aligned}
$$

As already said, the key property of quantum trajectory theory is that $p(t)$ is a mean-1 $\mathbb{Q}$ martingale, which follows from this equation and the normalization (4) of the initial condition [30, Theorem 2.4, Sec. 3.1].

a. The physical probability. Now we introduce the new probability measures, whose physical meaning will be discussed in Sec. III $\forall A \in \mathscr{F}_{T}$,

$$
\mathbb{P}_{\phi_{0}}^{T}(A):=\mathbb{E}_{\mathbb{Q}}\left[p(T) 1_{A}\right]=\int_{A} p(T ; \omega) \mathbb{Q}(\mathrm{d} \omega) .
$$

Owing to the martingale property of the probability density $p(t)$, the probabilities $\mathbb{P}_{\phi_{0}}^{T}$ are consistent, in the sense that $\mathbb{P}_{\phi_{0}}^{t}(F)=\mathbb{P}_{\phi_{0}}^{s}(F)$ for $F \in \mathscr{F}_{s}, t \geq s \geq 0$.

The new probability $\mathbb{P}_{\phi_{0}}^{T}$ modifies the distribution of the processes $B_{i}$ and $N_{k}$. A very important property is that a Girsanov-type theorem holds [30, Proposition 2.5, Remarks 2.6 and 3.5].

b. Girsanov transformation. Under $\mathbb{P}_{\phi_{0}}^{T}$, in the time interval $[0, T]$, the processes

$$
W_{j}(t):=B_{j}(t)-\int_{0}^{t} m_{j}(s) \mathrm{d} s, \quad j=1, \ldots, d,
$$

are independent Wiener processes, while the counting processes $N_{1}, \ldots, N_{d^{\prime}}$ change their stochastic intensities, which become $j_{1}, \ldots, j_{d^{\prime}}$. The quantities $m_{i}$ and $j_{k}$ are defined in Eqs. (11) and (12).

Note that, if for a certain index $i$ we have $m_{i}(t)=0$, $\forall t \geq 0$, then $W_{i}(t)=B_{i}(t)$ : the process $B_{i}$ remains a Wiener process also after the change of probability and it is independent from all the other components of $W$. For instance, from Eq. (11) we have $m_{i} \equiv 0$ for all initial conditions when the operator $\mathrm{i} L_{i}(t)$ is self-adjoint for all $t \geq 0$. 
c. The nonlinear SSE. Under $\mathbb{P}_{\phi_{0}}^{T}$, in the time interval $[0, T]$, the random normalized vector (10) satisfies the stochastic differential equation

$$
\begin{aligned}
\mathrm{d} \psi(t)= & \hat{K}(t) \psi\left(t_{-}\right) \mathrm{d} t \\
& +\sum_{i=1}^{d}\left(L_{i}(t)-\frac{1}{2} m_{i}(t)\right) \psi\left(t_{-}\right) \mathrm{d} W_{i}(t) \\
& +\sum_{k=1}^{d^{\prime}}\left(\frac{R_{k}(t) \psi\left(t_{-}\right)}{\left\|R_{k}(t) \psi\left(t_{-}\right)\right\|}-\psi\left(t_{-}\right)\right) \mathrm{d} N_{k}(t),
\end{aligned}
$$

with $\psi(0)=\phi_{0}$, and

$$
\begin{aligned}
& \hat{K}(t):=-\mathrm{i} H(t)-\frac{1}{2} \sum_{i=1}^{d}\left(L_{i}(t)^{\dagger}-m_{i}(t)\right) L_{i}(t) \\
& -\sum_{i=1}^{d} \frac{m_{i}(t)^{2}}{8}+\frac{1}{2} \sum_{k=1}^{d^{\prime}}\left(j_{k}(t)-i_{k}(t) R_{k}(t)^{\dagger} R_{k}(t)\right) .
\end{aligned}
$$

To get this result one needs to compute $\mathrm{d}(1 / \sqrt{p(t)})$ from Eq. (13) and to express this differential and $\mathrm{d} \phi(t)$ in terms of the new Wiener processes; the rigorous proof is given in Ref. [30].

At least in the Markov case, it is this equation that is the starting point for powerful numerical methods [1, 12].

\section{THE STOCHASTIC MASTER EQUATION}

Now that we have presented the theory of the stochastic Schrödinger equation for pure states, we develop the analog for density matrices and we introduce the stochastic master equation.

\section{A. The linear SME}

As in the case of the SSE, we start with a linear equation. More precisely, from Eqs. (5) and (8) we can derive the linear SME for the process $\tilde{\sigma}(t):=|\phi(t)\rangle\langle\phi(t)|, t \geq 0$ :

$$
\begin{array}{r}
\mathrm{d} \tilde{\sigma}(t)=\mathcal{L}(t)\left[\tilde{\sigma}\left(t_{-}\right)\right] \mathrm{d} t+\sum_{i=1}^{d}\left(L_{i}(t) \tilde{\sigma}\left(t_{-}\right)\right. \\
\left.+\tilde{\sigma}\left(t_{-}\right) L_{i}(t)^{\dagger}\right) \mathrm{d} B_{i}(t)+\sum_{k=1}^{d^{\prime}}\left(R_{k}(t) \tilde{\sigma}\left(t_{-}\right) R_{k}(t)^{\dagger}\right. \\
\left.-\tilde{\sigma}\left(t_{-}\right)\right)\left(\mathrm{d} N_{k}(t)-i_{k}(t) \mathrm{d} t\right),
\end{array}
$$

where $\mathcal{L}(t)$ is the following Liouville operator:

$$
\begin{gathered}
\mathcal{L}(t)[\tau]:=-\mathrm{i}[H(t), \tau]-\frac{1}{2} \sum_{i=1}^{d}\left\{L_{i}(t)^{\dagger} L_{i}(t), \tau\right\} \\
-\frac{1}{2} \sum_{k=1}^{d^{\prime}} i_{k}(t)\left\{R_{k}(t)^{\dagger} R_{k}(t), \tau\right\} \\
+\sum_{i=1}^{d} L_{i}(t) \tau L_{i}(t)^{\dagger}+\sum_{k=1}^{d^{\prime}} i_{k}(t) R_{k}(t) \tau R_{k}(t)^{\dagger} .
\end{gathered}
$$

Let us stress that this operator is random. In particular, this makes the solution $\tilde{\sigma}(t)$ non-Markovian since the randomness of the operator $\mathcal{L}(t)$ introduces a dependence on the past. This fact will be made explicit in the concrete model developed in Sec. IV

Let us note that the usual master equations (without the driving noises $B$ and $N$ ), but with stochastic Liouville operators, have already been considered in the literature as models of non-Markovian evolutions. Moreover, these equations have been derived from unitary systemenvironment dynamics by various techniques and approximations; see, for instance, [35].

\section{B. The nonlinear SME}

Note that the probability density (9) of $\mathbb{P}_{\phi_{0}}^{t}$ with respect to $\mathbb{Q}$ can be written as $p(t)=\operatorname{Tr}\{\tilde{\sigma}(t)\}$. Then, we normalize $\tilde{\sigma}(t)$ by defining the state $\tilde{\rho}(t)=$ $\tilde{\sigma}(t) / \operatorname{Tr}\{\tilde{\sigma}(t)\}$; when the denominator vanishes we take for $\tilde{\rho}(t)$ an arbitrary state. It is then possible to show that $\tilde{\rho}(t)$ satisfies the nonlinear SME under the new probability $\mathbb{P}_{\phi_{0}}^{T} \underline{30}$, Remark 3.6]:

$$
\begin{aligned}
\mathrm{d} \tilde{\rho}(t)= & \mathcal{L}(t)\left[\tilde{\rho}\left(t_{-}\right)\right] \mathrm{d} t+\sum_{i=1}^{d}\left(L_{i}(t) \tilde{\rho}\left(t_{-}\right)\right. \\
& \left.+\tilde{\rho}\left(t_{-}\right) L_{i}(t)^{\dagger}-m_{i}(t) \tilde{\rho}\left(t_{-}\right)\right) \mathrm{d} W_{i}(t) \\
+ & \sum_{k=1}^{d^{\prime}}\left(\frac{R_{k}(t) \tilde{\rho}\left(t_{-}\right) R_{k}(t)^{\dagger}}{\operatorname{Tr}\left\{R_{k}(t)^{\dagger} R_{k}(t) \tilde{\rho}\left(t_{-}\right)\right\}}-\tilde{\rho}\left(t_{-}\right)\right) \\
& \times\left(\mathrm{d} N_{k}(t)-j_{k}(t) \mathrm{d} t\right) .
\end{aligned}
$$

Everything can be expressed in terms of density matrices as we can write

$$
\begin{gathered}
m_{i}(t)=2 \operatorname{Re} \operatorname{Tr}\left\{L_{i}(t) \tilde{\rho}\left(t_{-}\right)\right\}, \\
j_{k}(t)=i_{k}(t) \operatorname{Tr}\left\{R_{k}(t)^{\dagger} R_{k}(t) \tilde{\rho}\left(t_{-}\right)\right\} .
\end{gathered}
$$

The nonlinear SME for $\tilde{\rho}(t)$ can also be directly obtained from (16) by remarking that

$$
\tilde{\rho}(t)=|\psi(t)\rangle\langle\psi(t)| \text {. }
$$




\section{The a priori states and the mean evolution}

The mean state, or a priori state, is defined by

$$
\eta(t):=\mathbb{E}_{\mathbb{Q}}[\tilde{\sigma}(t)] \equiv \mathbb{E}_{\mathbb{P}_{\phi_{0}}^{T}}[\tilde{\rho}(t)] .
$$

By Eqs. (18) and (20) one obtains

$$
\dot{\eta}(t)=\mathbb{E}_{\mathbb{Q}}[\mathcal{L}(t)[\tilde{\sigma}(t)]] \equiv \mathbb{E}_{\mathbb{P}_{\phi_{0}}^{T}}[\mathcal{L}(t)[\tilde{\rho}(t)]]
$$

A major difference with the usual Markovian situation is that in our case this equation is not closed. In the Markovian case one obtains an equation of the form $\dot{\eta}(t)=\mathcal{L}(t)[\eta(t)]$, but in our situation this is not possible, since the operator $\mathcal{L}(t)$ is random and contributes to the mean. Formally, a closed equation can be obtained by using projection techniques such as the Nakajima-Zwanzig method. This construction has been derived in [29], but the final equation is essentially not tractable.

It is then clear that the mean evolution is highly nonMarkovian. It is important to notice that our approach ensures that this evolution stays completely positive. We then obtain a completely positive non-Markovian behavior, the memory effect being encoded into the random Liouville operator $\mathcal{L}(t)$. In particular when $\mathcal{L}(t)$ is not random, we recover the usual Markovian framework.

\section{Measurement interpretation}

In this section, we present the essential ingredients needed in order to describe the measurement interpretation of our theory.

\section{Observed outputs}

Let us consider $a_{\ell j}(t, s), \quad \ell=1, \ldots, m_{I}, n_{h k}(t, s)$, $h=1, \ldots, m_{J}$, which are adapted and càglàd kernels and $e_{\ell}(t), w_{k}(t)$ which are adapted and càdlàg processes. We can then define the following processes, which represent the outputs of the continuous measurement process:

$$
\begin{aligned}
I_{\ell}(t) & :=\sum_{j=1}^{m} \int_{0}^{t} a_{\ell j}(t, s) \mathrm{d} M_{j}(s)+e_{\ell}(t), \\
J_{h}(t) & :=\sum_{k=1}^{d^{\prime}} \int_{(0, t]} n_{h k}(t, s) \mathrm{d} N_{k}(s)+w_{k}(t) .
\end{aligned}
$$

The idea underlying the construction of these processes is that the instantaneous outputs are the formal derivatives $\dot{M}_{j}(t)$ and $\dot{N}_{k}(t)$. The measuring apparatuses have a smoothing effect on the singular instantaneous outputs and can also provide some post-measurement processing of the outputs. These effects are represented by the integrals with the detector response functions $a_{\ell j}$ and $n_{h k}$.
Moreover, it is possible that the detectors introduce some further noise, for instance of electronic origin, and this is taken into account by the additive noises $e_{\ell}$ and $w_{k}$ and by the fact that response functions can be random.

Let us consider now all the events that can be observed up to time $t$, that is, the events determined by the outputs $I_{\ell}, J_{h}$ up to $t$. Let us denote by $\mathscr{G}_{t}$ the collection of such events. In mathematical terms $\mathscr{G}_{t}$ is the $\sigma$-algebra generated by $I_{\ell}(s)$ and $J_{h}(s)$, with $s \in[0, t]$, $\ell=1, \ldots, m_{I}, h=1, \ldots, m_{J}$. Because all the processes involved in the definition of the outputs are $\left(\mathscr{F}_{t}\right)$ adapted, we get $\mathscr{G}_{t} \subset \mathscr{F}_{t}$, for all $t$. Let us stress that in general we do not have $\mathscr{G}_{t}=\mathscr{F}_{t}$, because $\mathscr{G}_{t}$ contains only events that can be observed by the measuring apparatuses, while $\mathscr{F}_{t}$ can contain extra sources of noise, which can affect the system (a noisy environment for instance).

\section{Feedback}

In this formalism we can describe also measurementbased feedback: parts of the outputs are used to control some features of the dynamics or of the measuring apparatus, say through a stimulating laser or through a local oscillator in a homo- or heterodyne detector. When the feedback involves the output in the past, other memory effects are introduced. A typical measurement-based feedback is represented by a Hamiltonian term functionally dependent on some output up to the current time; while in this way it becomes a random Hamiltonian, its contribution is perfectly compatible with the whole formalism. We shall not give examples in this paper; the theory and some applications can be found in 30, Sec. 4.4] and [32].

\section{Instruments and a posteriori state}

A cornerstone of a consistent measurement interpretation of SME relies on the introduction of the so-called instruments. In order to develop this theory we need to define the propagator $\mathcal{A}(t, s)$ of Eq. (18), that is, the random linear map $\tilde{\sigma}(s) \mapsto \tilde{\sigma}(t)$. An essential point is that this application is completely positive and satisfies the composition rule $\mathcal{A}(t, s)=\mathcal{A}(t, r) \circ \mathcal{A}(r, s), 0 \leq s \leq r \leq t$.

Now for an event $A \in \mathscr{G}_{t}$, we define

$$
\mathcal{I}_{t}(A)[\rho]=\mathbb{E}_{\mathbb{Q}}\left[\mathbf{1}_{A} \mathcal{A}(t, 0)[\rho]\right] .
$$

For all $A \in \mathscr{G}_{t}, \mathcal{I}_{t}(A)$ is a completely positive linear map called an instrument. In particular this gives the probability that an event $A \in \mathscr{G}_{t}$ occurs. More precisely, if $\rho_{0}$ represents the pre-measurement state, the probability of $A \in \mathscr{G}_{t}$ is given by

$$
\operatorname{Tr}\left\{\mathcal{I}_{t}(A)\left[\rho_{0}\right]\right\}=\mathbb{P}_{\phi_{0}}^{t}[A], \quad \mathbb{E}_{\mathbb{Q}}\left[\left|\phi_{0}\right\rangle\left\langle\phi_{0}\right|\right]=\rho_{0},
$$

and we recover the previous definition of the physical probability. 
Then, we can define the a posteriori state by

$$
\rho(t):=\mathbb{E}_{\mathbb{P}_{\phi_{0}}^{T}}\left[\tilde{\rho}(t) \mid \mathscr{G}_{t}\right] \equiv \frac{\sigma(t)}{\operatorname{Tr}\{\sigma(t)\}},
$$

where

$$
\sigma(t):=\mathbb{E}_{\mathbb{Q}}\left[\tilde{\sigma}(t) \mid \mathscr{G}_{t}\right]
$$

The state $\rho(t)$ corresponds to the update of the state of the system conditionally on the observation of the outputs up to time $t$.

It is important to notice that in general we can not derive a closed equation for $\rho(t)$ such as the one for $\tilde{\rho}(t)$. Essentially, it depends whether or not $\mathscr{G}_{t}=\mathscr{F}_{t}$. In the case of $\mathscr{G}_{t} \neq \mathscr{F}_{t}$ the randomness of the operators appearing in $\mathcal{L}(t)$ will prevent the equation from being closed; again some projection technique could be used to obtain a kind of closed equation, but it would be intractable for practical purposes.

As a conclusion, we can see that this approach allows us to describe non-Markovian evolutions that are generalizations of the Markovian setup. As we shall see, the randomness of the operators $L_{i}(t)$ and $R_{k}(t)$ will be used to describe concrete non-Markovian effects such as colored environments and incoherent stimulating light.

The physical model is determined by the physical probability, the nonlinear SME, and the outputs, not by the SSE, which is not unique. Two SSEs giving solutions that differ only by a stochastic phase are physically equivalent; no physical consequence depends on a global phase in $\phi(t)$ or $\psi(t)$ [14, Sec. 2.5].

\section{A MODEL: A NOISY OSCILLATOR}

Let us present now a mathematically treatable but sufficiently rich and physically interesting model; the aim is to understand what kind of physical phenomena and memory effects can be described by the theory we have presented. To be simple we take a linear system, but we allow for absorption, emission, colored noises acting on the system and on the detection apparatuses, and so on. The general scheme is the following:

1. The quantum system is a single oscillator; to fix the ideas we think of a mode in an optical cavity, but it could be an ion in a trap or some other system in the harmonic approximation. Let $a$ and $a^{\dagger}$ be the usual annihilation and creation operators of quanta in the mode; then, the free Hamiltonian of the oscillator is

$$
H_{0}(t) \equiv H_{0}=\nu_{0} a^{\dagger} a, \quad \nu_{0}>0 .
$$

2. The system emits and absorbs light; the systemelectromagnetic-field interaction is treated in the usual Markov approximation. (a) Some emitted light reaches a photocounter: direct detection. The post-processing of the output is taken into account by a detector response function.

(b) Some light reaches a homo- or heterodyne detector. The function describing the local oscillator can be random, a way to model imperfections. We shall show that this fact introduces memory in the detection process, not in the mean dynamics. Moreover, we can have also a detector response function acting as a frequency filter; see Eq. (91).

(c) We introduce a stimulating laser; the laserwave-oscillator interaction is treated in the usual dipole and rotating-wave approximations. The laser wave can be random because the laser is noisy and/or because of feedback. This introduces memory also into the Liouvillian and in the mean dynamics, in spite of the fact that the interaction is without memory.

3. We introduce various kinds of colored environments. According to the choices of the parameters these new terms can describe incoherent light, a squeezed reservoir, a usual (or colored) thermal bath, intermediate situations, and so on.

As in the general part, also in this model $B_{1}, \ldots, B_{d}$ are $d$ independent standard Wiener processes under the reference probability $\mathbb{Q}$; here we shall have $d \geq 5$. Moreover, we shall introduce $m=5$ diffusive channels and a single jump channel, $d^{\prime}=1$. Finally, we shall introduce a single diffusive output and a single counting output; according to the notations of Sec. IIID 1 we shall have $m_{I}=m_{J}=1$.

\section{A. Stimulating laser and emitted light detection}

As already said we consider the oscillatorelectromagnetic-field interaction in the dipole and rotating-wave approximations. We divide the directions of the propagating light into some "channels". The index 1 labels the "side" channels used to describe the emitted light reaching a photo-counter (direct detection) or a homo- or heterodyne detector. Channels 2 and 3 are the "forward" channels in the direction of the stimulating laser; they describe also losses of light.

\section{Detection}

a. Direct detection. We consider only one counter, so that we have $d^{\prime}=1$. Under the reference probability $\mathbb{Q}$, the associated counting process $N_{1}(t) \equiv N(t)$ is taken to be a Poisson process of intensity $i_{1}(t) \equiv \lambda \geq 0$. When $\lambda=0$ this channel is not open. The associated operator 
is

$$
R_{1}(t) \equiv R=\bar{\beta} a, \quad \beta \in \mathbb{C} .
$$

With respect to the general case of Sec. IIID1, let us consider only deterministic, time invariant, real and continuous detector response functions, so that we have $J_{1}(t) \equiv J(t), n_{11}(t, s)=F_{J}(t-s), w_{1}(t)=0$, and the output current is

$$
J(t)=\int_{0}^{t} F_{J}(t-r) \mathrm{d} N(r) .
$$

b. Homodyne or heterodyne detection. As usual, homo- or heterodyne detection is described in the Markov approximation by a diffusive channel driven by a Wiener process $B_{1}$ (under the reference probability $\mathbb{Q}$ ) [14, Sec. 7.2]. By particularizing the quantities introduced in Sec. IIA we have $L_{1}^{0}(t)=L_{1}(t)$ and $M_{1}(t)=B_{1}(t)$, which means $f_{1}(t)=0$ and $b_{1 i}(t)=\delta_{1 i}$. Then we take

$$
L_{1}(t)=-\mathrm{i} \overline{\alpha_{1}} \overline{h(t)} a, \quad|h(t)|=1, \quad \alpha_{1} \in \mathbb{C} ;
$$

$h(t)$ is the contribution of the local oscillator, which can be random. Randomness in the local oscillator can be due to imperfections, but it could be due also to the fact that $h(t)$ is taken dependent on some of the observed outputs at previous times in order to describe adaptive measurements, as is done in [15, Sec. 7.9.2].

We consider again a deterministic, time invariant, real and continuous response function $F_{I}$; in terms of the notation of Sec. IIID1 we take $I_{1}(t)=I(t), a_{1 j}(t, s)=$ $F_{I}(t-s)$, and $e_{1}(t)=0$. Then, the output current of the homo- or heterodyne detector is

$$
I(t)=\int_{0}^{t} F_{I}(t-r) \mathrm{d} B_{1}(r)
$$

We assume the response function $F_{I}$ to be in $L^{1}\left(\mathbb{R}_{+}\right)$, so that its Fourier transform exists:

$$
G_{I}(\mu):=\int_{0}^{+\infty} \mathrm{e}^{\mathrm{i} \mu t} F_{I}(t) \mathrm{d} t .
$$

We shall see in Sec. IVE that $\left|G_{I}(\mu)\right|^{2}$ has the role of a linear frequency filter on the output.

c. Contribution to the linear SSE. Summarizing, the contributions to the right hand side of the linear SSE (11) or (5) of the two detection channels are

$$
\begin{array}{r}
\frac{\lambda}{2}\left(\mathbb{1}-|\beta|^{2} a^{\dagger} a\right) \phi\left(t_{-}\right) \mathrm{d} t+(\bar{\beta} a-\mathbb{1}) \phi\left(t_{-}\right) \mathrm{d} N(t) \\
-\frac{\left|\alpha_{1}\right|^{2}}{2} a^{\dagger} a \phi\left(t_{-}\right) \mathrm{d} t-\mathrm{i} \overline{\alpha_{1}} \overline{h(t)} a \phi\left(t_{-}\right) \mathrm{d} B_{1}(t) .
\end{array}
$$

The final linear SSE is given by Eq. (59).

\section{The forward channels}

Channels 2 and 3 represent the forward channel (the direction of the stimulating laser) and the lost light; we can include in these channels other Markovian dissipative contributions. There is no detector associated with these channels, and we choose to put a diffusive component (the Wiener $B_{2}$ ) in channel 2, while channel 3 is used to complete the Hamiltonian part with the contribution of the stimulating laser. With respect to the symbols used in the linear SSE (11) and in Sec. II A we take

$$
L_{2}^{0}(t) \equiv L_{2}^{0}=-\mathrm{i} \overline{\alpha_{2}} a, \quad L_{3}^{0}(t) \equiv L_{3}^{0}=-\mathrm{i} \alpha_{2} a^{\dagger},
$$

with $\alpha_{2} \in \mathbb{C}$, and $\overline{f_{2}(t)}=f_{3}(t)=f(t), b_{2 i}(t)=\delta_{2 i}$, $b_{3 i}(t)=0$, which give $L_{2}(t)=L_{2}^{0}, L_{3}(t)=0$,

$$
\mathrm{d} M_{2}(t)=\overline{f(t)} \mathrm{d} t+\mathrm{d} B_{2}(t), \quad \mathrm{d} M_{3}(t)=f(t) \mathrm{d} t .
$$

a. Contribution to the linear SSE. Summarizing, the contributions to the right-hand side of the linear SSE (5) of channels 2 and 3 are

$$
\left(-\frac{\left|\alpha_{2}\right|^{2}}{2} a^{\dagger} a-\mathrm{i} H_{f}(t)\right) \phi\left(t_{-}\right) \mathrm{d} t-\mathrm{i} \overline{\alpha_{2}} a \phi\left(t_{-}\right) \mathrm{d} B_{2}(t),
$$

where $H_{f}(t)$ contains the interaction between the stimulating external laser and the oscillator:

$$
H_{f}(t)=\overline{\alpha_{2}} \overline{f(t)} a+\alpha_{2} f(t) a^{\dagger} .
$$

b. Stimulating laser. The function $f(t)$ represents the laser wave, eventually a laser with imperfections [14]. In the case of closed loop control, the laser wave could depend on the observed output [32], but here we disregard the possibility of feedback. Then a good model for a not perfectly coherent stimulating laser is the phase diffusion model [36]. Let $\nu_{3}>0$ be the carrier frequency of the laser light (in this case $\Delta \nu=\nu_{0}-\nu_{3}$ is called the detuning) and let $\varepsilon>0$ be its bandwidth; then

$$
f(t)=g \exp \left\{-\mathrm{i} \nu_{3} t+\mathrm{i} \sqrt{\varepsilon} B_{3}(t)\right\}, \quad g \in \mathbb{C} .
$$

The quantity $g$ contains the amplitude and the initial phase of the laser; in principle it could be a random variable, but for simplicity here we take it to be deterministic.

To identify the bandwidth of the laser light $f$, we consider its spectrum. Since $f$ is a complex stochastic process, its spectrum is given by the classical definition [37]

$$
S_{f}(\mu):=\lim _{T \rightarrow+\infty} \frac{1}{T} \mathbb{E}_{\mathbb{Q}}\left[\left|\int_{0}^{T} \mathrm{e}^{\mathrm{i} \mu t} f(t) \mathrm{d} t\right|^{2}\right] .
$$

By using the autocorrelation function (A2) of the process $f$ we easily get the Lorentzian spectrum

$$
S_{f}(\mu)=\frac{\varepsilon|g|^{2}}{\left(\mu-\nu_{3}\right)^{2}+\varepsilon^{2} / 4} .
$$


c. Homodyne detection. In this case the local oscillator and the stimulating light are generated by the same laser. A choice, that takes into account the differences in the optical paths, is

$$
h(t)=\mathrm{e}^{\mathrm{i} \theta} \frac{f(t-\Delta t)}{|f(t-\Delta t)|}, \quad \theta \in \mathbb{R} ;
$$

we are assuming $f(t) \neq 0$. The phase $\theta$ and the time shift $\Delta t$ depend on the physical implementation of the homodyne apparatus and could be random, but, for simplicity, we take both to be deterministic, $\theta \in[0,2 \pi)$ and $\Delta t \in \mathbb{R}$.

d. Heterodyne detection. The local oscillator $h(t)$ and the stimulating wave $f(t)$ are produced by different laser sources and the phase difference is not stable; the carrier frequencies are generally different. In this case $h$ could depend on the output (another form of closed loop control) or could be described by a phase diffusion model (noise in the local oscillator). In this second case we can take

$$
h(t)=\exp \left\{\mathrm{i} \vartheta-\mathrm{i} \nu t+\mathrm{i} \sqrt{\kappa} B_{4}(t)\right\},
$$

$\nu \in \mathbb{R}, \kappa>0, \vartheta \in \mathbb{R}$.

\section{Summary of the contributions to the linear SME}

We have already explicitly given the various contributions to the SSE. To understand better the meaning of these terms it is worthwhile to write down how they contribute to the linear SME (18) and to the random Liouville operator (19). Let us consider the free Hamiltonian of the oscillator and all the other terms we have introduced up to now; let us set

$$
\gamma_{0}:=\left|\alpha_{1}\right|^{2}+\left|\alpha_{2}\right|^{2}+|\beta|^{2} \lambda>0
$$

and $\tilde{\sigma}(t):=|\phi(t)\rangle\langle\phi(t)|$ as in Sec. III A Then, we have

$$
\begin{aligned}
\mathrm{d} \tilde{\sigma}(t) & =\mathcal{L}_{\mathrm{em}}(t)\left[\tilde{\sigma}\left(t_{-}\right)\right] \mathrm{d} t \\
+ & \left(|\beta|^{2} a \tilde{\sigma}\left(t_{-}\right) a^{\dagger}-\tilde{\sigma}\left(t_{-}\right)\right)(\mathrm{d} N(t)-\lambda \mathrm{d} t) \\
+ & \left(\mathrm{i} \alpha_{1} h(t) \tilde{\sigma}\left(t_{-}\right) a^{\dagger}-\mathrm{i} \overline{\alpha_{1}} \overline{h(t)} a \tilde{\sigma}\left(t_{-}\right)\right) \mathrm{d} B_{1}(t) \\
& +\left(\mathrm{i} \alpha_{2} \tilde{\sigma}\left(t_{-}\right) a^{\dagger}-\mathrm{i} \overline{\alpha_{2}} a \tilde{\sigma}\left(t_{-}\right)\right) \mathrm{d} B_{2}(t)+\cdots
\end{aligned}
$$

(the ellipsis stands for further contributions that we shall introduce in Sec. IVB),

$$
\begin{aligned}
\mathcal{L}_{\mathrm{em}}(t)[\tau]=-\mathrm{i}\left[H_{0}+\right. & \left.H_{f}(t), \tau\right] \\
& +\gamma_{0} a \tau a^{\dagger}-\frac{\gamma_{0}}{2}\left\{a^{\dagger} a, \tau\right\} .
\end{aligned}
$$

From these equations it is apparent that the electromagnetic interaction has been treated in the usual Markov approximation; we see also that the parameter $\gamma_{0}$ is the mode width. The only possible sources of memory are $f$ (the stimulating laser light) and $h$ (the local oscillator). So, up to now the memory is due only to the imperfections inducing randomness in the lasers involved. Remember that we have not included a conceptually very important source of memory, the possibility of feedback.

\section{B. A colored environment}

Our aim here is to introduce some sources of colored noise; they could describe physically different scenarios, which we shall discuss at the end of the section.

Let us introduce a complex Gaussian process $Y$ given by

$$
\begin{gathered}
Y(t):=\sum_{j=5}^{d}\left(b_{j} B_{j}(t)+\int_{0}^{t} X_{j}(s) \mathrm{d} s\right), \\
X_{j}(s)=\int_{0}^{s} c_{j}(s-u) \mathrm{d} B_{j}(u) .
\end{gathered}
$$

Here $b_{j} \in \mathbb{C}$ and we set

$$
q:=\sum_{j=5}^{d} b_{j}{ }^{2}, \quad k:=\sum_{j=5}^{d}\left|b_{j}\right|^{2}
$$

moreover, we assume the complex functions $c_{j}$ to be integrable, i.e.

$$
\int_{0}^{+\infty}\left|c_{j}(t)\right| \mathrm{d} t<+\infty .
$$

Now, we add two more diffusive channels; with the notations of Sec. III, we take $m=5$ and

$$
\begin{gathered}
L_{4}^{0}(t) \equiv L_{4}^{0}=-\mathrm{i} a, \quad L_{5}^{0}(t) \equiv L_{5}^{0}=-\mathrm{i} a^{\dagger}, \\
M_{4}(t)=\overline{Y(t)}, \quad M_{5}(t)=Y(t),
\end{gathered}
$$

that means $\overline{f_{4}(t)}=f_{5}(t)=\sum_{j=5}^{d} X_{j}(t)$,

$$
\overline{b_{4 i}(t)}=b_{5 i}(t)=\left\{\begin{array}{ll}
0, & i \leq 4 \\
b_{i}, & i \geq 5
\end{array} .\right.
$$

This gives $L_{4}(t)=0$ and, for $i \geq 5$,

$$
L_{i}(t) \equiv L_{i}=\overline{b_{i}} L_{4}^{0}+b_{i} L_{5}^{0}=-\mathrm{i}\left(\overline{b_{i}} a+b_{i} a^{\dagger}\right) .
$$

The contribution of these new terms to the linear SSE (11) turns out to be

$$
-\frac{D}{2} \phi\left(t_{-}\right) \mathrm{d} t-\mathrm{i}\left(a^{\dagger} \mathrm{d} Y(t)+a \mathrm{~d} \overline{Y(t)}\right) \phi\left(t_{-}\right)
$$

where

$$
D:=2 k a^{\dagger} a+k+\bar{q} a^{2}+q a^{\dagger^{2}} .
$$




\section{The spectrum of the Gaussian noise}

The dynamics of our system involves the differential of the process $Y$ or, in other terms, its generalized derivative $\dot{Y}(t)$. Like the spectrum of $f(\underline{36})$, the spectrum of this classical complex process is defined by

$$
S_{Y}(\mu):=\lim _{T \rightarrow+\infty} \frac{1}{T} \mathbb{E}_{\mathbb{Q}}\left[\left.|| \int_{0}^{T} \mathrm{e}^{\mathrm{i} \mu t} \mathrm{~d} Y(t)\right|^{2}\right]
$$

when the limit exists.

By construction, $Y$ is a Gaussian process with zero mean; its second moments, needed in (52), can be easily computed by using the properties of the stochastic integrals (the Itô isometry).

Let us introduce the Laplace transform of $c_{j}$

$$
s_{j}(z):=\int_{0}^{+\infty} \mathrm{e}^{-z t} c_{j}(t) \mathrm{d} t, \quad \operatorname{Re} z \geq 0,
$$

which exists owing to the integrability condition (46).

The spectrum (52) is computed in Appendix A2 and it is given by

$$
S_{Y}(\mu)=\sum_{j=5}^{d} S_{Y_{j}}(\mu), \quad S_{Y_{j}}(\mu)=\left|b_{j}+s_{j}(-\mathrm{i} \mu)\right|^{2} .
$$

Note that the spectrum of $Y(t)$ is the sum of the spectra of the components $Y_{j}(t)$ without any interference among them. Each spectral component contains a white-noise contribution $\left(b_{j}\right)$ and a regular one $\left(s_{j}\right)$, which interfere (they sum up inside the square modulus). Moreover, let us stress that by this construction it is possible to insert Gaussian noises with given spectra, not only in this model, but even in the general theory.

\section{Contribution to the linear SME}

As was done for the electromagnetic contributions in Eq. (41), it is useful to identify the contributions to the linear SME due to the new noises:

$$
\begin{aligned}
\mathrm{d} \tilde{\sigma}(t)=\cdots+\sum_{j=5}^{d}\left(\mathcal{L}_{j}(t)\left[\tilde{\sigma}\left(t_{-}\right)\right] \mathrm{d} t\right. & \\
& \left.-\mathrm{i}\left[C_{j}, \tilde{\sigma}\left(t_{-}\right)\right] \mathrm{d} B_{j}(t)\right)
\end{aligned}
$$

(the ellipsis stands for the contributions already introduced), where

$$
\begin{gathered}
\mathcal{L}_{j}(t)[\tau]:=-\mathrm{i}\left[H_{j}(t), \tau\right]-\frac{1}{2}\left[C_{j},\left[C_{j}, \tau\right]\right], \\
C_{j}:=b_{j} a^{\dagger}+\overline{b_{j}} a, \quad H_{j}(t):=X_{j}(t) a^{\dagger}+\overline{X_{j}(t)} a .
\end{gathered}
$$

Let us note that the contributions of the classical processes $X_{j}$ to the dynamics (55) are very reminiscent of the contribution of classical processes in the "adjoint equation" in [13, Sec. 3.5]. This shows that these contributions, or at least some of them, can come from the interaction of the system with a quantum reservoir and could be derived by using the techniques of the quantum Langevin equation and the adjoint equation [13, Secs. 3.1 and 3.5].

Now we can identify the physical meaning of various possible contributions.

a. Incoherent light. Consider the index $j=5$ and assume $b_{5}=0$. Then, $C_{5}=0$ and this term contributes only with a regular random Hamiltonian term $H_{5}(t)$. Its structure is very similar to that of $H_{f}(t)$, but the two random processes involved are qualitatively different. The process $f$ is the exponential of a Gaussian process and represents a quasi-monochromatic wave (laser light). The process $X_{5}$ is Gaussian and could represent, for instance, incoherent light with an arbitrary spectrum $\left|s_{5}(-\mathrm{i} \mu)\right|^{2}$, for instance, thermal light with a black-body spectrum [2, Eqs. (1.52) and (7.148)]

$$
\left|s_{5}(-\mathrm{i} \mu)\right|^{2}=\frac{6 \hbar^{2} \mu \mathrm{e}^{-\hbar \mu / k_{B} T}}{\pi^{2} k_{B}^{2} T^{2}\left(1-\mathrm{e}^{-\hbar \mu / k_{B} T}\right)}, \quad \mu>0 .
$$

Another possible choice for incoherent light is an Ornstein-Uhlenbeck process, that means taking

$$
c_{5}(t)=1_{(0,+\infty)}(t) g_{5} \mathrm{e}^{-\overline{\varkappa_{5}} t}, \quad \varkappa_{5}=\frac{\gamma_{5}}{2}-\mathrm{i} \nu_{5},
$$

with $g_{5} \in \mathbb{C}, \nu_{5} \in \mathbb{R}, \gamma_{5}>0$. In this case, from (53) we get

$$
s_{5}(z)=\frac{g_{5}}{z+\overline{\varkappa_{5}}}
$$

and the contribution to the spectrum (54) is the Lorentzian term

$$
\left|s_{5}(-\mathrm{i} \mu)\right|^{2}=\frac{\left|g_{5}\right|^{2}}{\left(\mu-\nu_{5}\right)^{2}+\gamma_{5}^{2} / 4} .
$$

As already seen, also the phase diffusion model (35) of the laser gives a Lorentzian spectrum (37), but, in spite of this, the two cases are completely different. The wave (35) is quasi-coherent, while the Ornstein-Uhlenbeck process represents a Gaussian incoherent wave.

b. Squeezed reservoir. Consider now the indices $j=$ 6,7 and assume $c_{6}=c_{7}=0$; then, we get $X_{6}=X_{7}=$ 0 and the contributions of these terms are Markovian. Indeed, by defining

$$
\begin{gathered}
n:=\frac{\left|b_{6}\right|^{2}+\left|b_{7}\right|^{2}}{\gamma_{0}}, \quad m:=-\frac{b_{6}{ }^{2}+b_{7}{ }^{2}}{\gamma_{0}}, \\
\mathrm{~d} C(t):=\frac{b_{6} \mathrm{~d} B_{6}(t)+b_{7} \mathrm{~d} B_{7}(t)}{\sqrt{\left|b_{6}\right|^{2}+\left|b_{7}\right|^{2}}},
\end{gathered}
$$


we get

$$
\begin{aligned}
& \sum_{j=6}^{7} \mathcal{L}_{j}(t)[\tau]=\gamma_{0} n\left(a \tau a^{\dagger}+a^{\dagger} \tau a-\frac{1}{2}\left\{a^{\dagger} a\right.\right. \\
& \left.\left.+a a^{\dagger}, \tau\right\}\right)-\gamma_{0} m\left(a^{\dagger} \tau a^{\dagger}-\frac{1}{2}\left\{a^{\dagger} a^{\dagger}, \tau\right\}\right) \\
& -\gamma_{0} \bar{m}\left(a \tau a-\frac{1}{2}\{a a, \tau\}\right), \\
& -\mathrm{i} \sum_{j=6}^{7}\left[C_{j}, \tau\right] \mathrm{d} B_{j}(t) \\
& =\mathrm{i} \sqrt{\gamma_{0} n}\left([\tau, a] \mathrm{d} \overline{C(t)}-\left[a^{\dagger}, \tau\right] \mathrm{d} C(t)\right) .
\end{aligned}
$$

By combining these contributions with the dissipative term in $\mathcal{L}_{\text {em }}(t)$ (42) we get the typical dissipative effect of a squeezed reservoir [13, Eq. (10.2.42)]. So we can interpret $n$ as the effective photon number and $m$ as the squeezing parameter of the reservoir. When $m=0$, i.e. $b_{7}= \pm \mathrm{i} b_{6}$, the previous dissipative terms reduce to the contribution of a thermal bath with $\gamma_{0} n=2\left|b_{6}\right|^{2}$.

c. The generic case. The spectrum (54) shows that the generic case is in between the two cases discussed above: a Markovian dissipative contribution and a random Hamiltonian contribution with interference between them.

\section{The full model}

Putting together all the contributions we have introduced in Eqs. (26), (50) and in Sec. IV A 2a, we get that the full linear SSE can be written as

$$
\begin{aligned}
\mathrm{d} \phi(t)= & \left(-\overline{\varkappa_{0}} a^{\dagger} a+\frac{\lambda-D}{2}\right) \phi\left(t_{-}\right) \mathrm{d} t \\
& -\mathrm{i} a^{\dagger} \phi\left(t_{-}\right) \mathrm{d} Y_{1}(t)-\mathrm{i} a \phi\left(t_{-}\right) \mathrm{d} Y_{2}(t) \\
& +(\bar{\beta} a-\mathbb{1}) \phi\left(t_{-}\right) \mathrm{d} N(t),
\end{aligned}
$$

where $\lambda$ is the intensity of the Poisson process $N$,

$$
\begin{gathered}
\mathrm{d} Y_{1}(t)=\alpha_{2} f(t) \mathrm{d} t+\mathrm{d} Y(t), \\
\mathrm{d} Y_{2}(t)=\overline{\alpha_{1}} \overline{h(t)} \mathrm{d} B_{1}(t)+\overline{\alpha_{2}} \overline{f(t)} \mathrm{d} t \\
+\overline{\alpha_{2}} \mathrm{~d} B_{2}(t)+\mathrm{d} \overline{Y(t)}, \\
\varkappa_{0}=-\mathrm{i} \nu_{0}+\frac{\gamma_{0}}{2}, \quad|h(t)|=1 ;
\end{gathered}
$$

$\gamma_{0}, Y$, and $D$, are given by Eqs. (40), (43), and (51).
1. The Liouville operator and the linear $S M E$

From Eqs. (42) and (56) we get the full Liouville operator

$$
\begin{gathered}
\mathcal{L}(t)[\tau]=-\mathrm{i}[H(t), \tau]+\gamma_{0}\left(a \tau a^{\dagger}-\frac{1}{2}\left\{a^{\dagger} a, \tau\right\}\right) \\
-\frac{1}{2} \sum_{j=5}^{d}\left[b_{j} a^{\dagger}+\overline{b_{j}} a,\left[b_{j} a^{\dagger}+\overline{b_{j}} a, \tau\right]\right] \\
H(t)=H_{0}+\overline{\mathrm{f}(t)} a+\mathrm{f}(t) a^{\dagger} \\
\mathrm{f}(t)=\alpha_{2} f(t)+\sum_{j=5}^{d} X_{j}(t) .
\end{gathered}
$$

Note that the Liouville operator is random only because of the presence of the process $f(t)$, but this is enough to preclude having a closed master equation for the a priori states, see Sec. IIIC Let us stress again the different physical roles of the wave $f$ and the Gaussian processes $X_{j}$, as discussed in Sec. IV B 2a.

By putting together Eqs. (41) and (55) we get the full linear SME

$$
\begin{aligned}
\mathrm{d} \tilde{\sigma}(t)= & \mathcal{L}(t)\left[\tilde{\sigma}\left(t_{-}\right)\right] \mathrm{d} t-\mathrm{i} \sum_{i=5}^{d}\left[\overline{b_{i}} a+b_{i} a^{\dagger}, \tilde{\sigma}(t)\right] \mathrm{d} B_{i}(t) \\
- & \mathrm{i}\left(\overline{\alpha_{1}} \overline{h(t)} a \tilde{\sigma}(t)-\alpha_{1} h(t) \tilde{\sigma}(t) a^{\dagger}\right) \mathrm{d} B_{1}(t) \\
& -\mathrm{i}\left(\overline{\alpha_{2}} a \tilde{\sigma}(t)-\alpha_{2} \tilde{\sigma}(t) a^{\dagger}\right) \mathrm{d} B_{2}(t) \\
+ & \left(|\beta|^{2} a \tilde{\sigma}\left(t_{-}\right) a^{\dagger}-\tilde{\sigma}\left(t_{-}\right)\right)(\mathrm{d} N(t)-\lambda \mathrm{d} t) .
\end{aligned}
$$

In the measuring process there is one more source of memory coming in through the randomness in the local oscillator $h(t)$.

\section{The solution of the linear SSE}

The simplifying mathematical feature of the model we have constructed is that the SSE leaves invariant the coherent states. Such states are defined by $a e(\xi)=\xi e(\xi)$, $\|e(\xi)\|=1$. We assume the initial condition to be the coherent vector

$$
\phi_{0}=e\left(\xi_{0}\right), \quad \xi_{0} \in \mathbb{C}
$$

To find the solution of the SSE (159) we make the ansatz

$$
\phi(t)=V(t) \mathrm{e}^{\frac{1}{2} Z(t)} e(\xi(t))
$$

As $\|\phi(t)\|^{2} \equiv|V(t)|^{2} \mathrm{e}^{\operatorname{Re} Z(t)}$ must be a martingale, we ask 
- $V(t)$ to contain only the contributions from the jumps of $N(t)$ and to be such that $|V(t)|^{2}$ is a martingale,

- $Z(t)$ to contain only the contributions from the diffusive processes and to be such that $\mathrm{e}^{\operatorname{Re} Z(t)}$ is a martingale,

- $\xi(t)$ to be a generic stochastic process whose differential contains all the possible terms.

Then, we identify $\mathrm{d}\langle e(\epsilon) \mid \phi(t)\rangle$ with $\langle e(\epsilon) \mid \mathrm{d} \phi(t)\rangle$, where now $\mathrm{d} \phi(t)$ is taken from Eq. (59). By equating the coefficients of the monomials with homogeneous powers of $\epsilon$ we get stochastic equations for the unknown terms, which can be solved. The final result is

$$
\begin{aligned}
& \xi(t)=\mathrm{e}^{-\overline{\varkappa_{0}} t} \xi_{0}-\mathrm{i} U_{f}(t)-\mathrm{i} U_{Y}(t), \\
& U_{f}(t):=\alpha_{2} \int_{0}^{t} \mathrm{e}^{-\overline{\varkappa_{0}}(t-r)} f(r) \mathrm{d} r \\
& U_{Y}(t):=\sum_{j=5}^{d} \int_{0}^{t} g_{j}(t-r) \mathrm{d} B_{j}(r) \\
& g_{j}(t):=\mathrm{e}^{-\overline{\varkappa_{0}} t} b_{j}+\int_{0}^{t} \mathrm{e}^{-\overline{\varkappa_{0}}(t-u)} c_{j}(u) \mathrm{d} u \\
& Z(t)=-2 \mathrm{i} \int_{0}^{t} \xi(s)\left(\overline{\alpha_{1}} \overline{h(s)} \mathrm{d} B_{1}(s)+\overline{\alpha_{2}} \mathrm{~d} B_{2}(s)\right) \\
& -2 \mathrm{i} \operatorname{Re} \int_{0}^{t} \overline{\xi(s)} \mathrm{d} Y_{1}(s)+\int_{0}^{t}\left({\overline{\alpha_{1}}}^{2} \overline{h(s)}^{2}+{\overline{\alpha_{2}}}^{2}\right) \xi(s)^{2} \mathrm{~d} s \\
& -\left(\left|\alpha_{1}\right|^{2}+\left|\alpha_{2}\right|^{2}\right) \int_{0}^{t}|\xi(s)|^{2} \mathrm{~d} s \\
& V(t)=\exp \left\{\frac{\lambda}{2} \int_{0}^{t}\left(1-|\beta|^{2}|\xi(s)|^{2}\right) \mathrm{d} s\right\} \\
& \times \prod_{r \in(0, t]}[\bar{\beta} \xi(r)]^{\Delta N(r)} .
\end{aligned}
$$

Let us stress that, almost surely, the product in (70) contains a finite number of factors different from 1 and that the expression (70) is nothing but the solution of the linear SDE

$$
\begin{aligned}
\mathrm{d} V(t)=V(t)\left\{\frac{\lambda}{2}\left(1-|\beta|^{2}|\xi(t)|^{2}\right) \mathrm{d} t\right. & \\
& +(\bar{\beta} \xi(t)-1) \mathrm{d} N(t)\}
\end{aligned}
$$

with initial condition $V(0)=1$. Independently of the methods used to find the solution, by using stochastic calculus, one can check that the expression for $\phi(t)$ defined by (64)-(70) indeed solves the SSE (59).

Note that the processes $\xi(t)$ and $Z(t)$ are continuous in time and that the contribution of the jumps is concentrated in $V(t)$.

\section{The physical probability}

Let us recall that the new probability is $\mathbb{P}_{\phi_{0}}^{T}(\mathrm{~d} \omega)=$ $p(t, \omega) \mathbb{Q}(\mathrm{d} \omega)$ (14). Having the explicit form of the solution of the linear SSE, we can compute the probability density $p(t)$ (9) and the normalized vector (10), that solves the nonlinear SSE (16). From Eqs. (9)-(12) we get

$$
\begin{gathered}
p(t)=\|\phi(t)\|^{2}=|V(t)|^{2} \mathrm{e}^{\operatorname{Re} Z(t)} \\
\psi(t)=\exp \left\{\mathrm{i} \arg V(t)+\frac{\mathrm{i}}{2} \operatorname{Im} Z(t)\right\} e(\xi(t)), \\
|V(t)|^{2}=\exp \left\{\int_{0}^{t}(\lambda-j(s)) \mathrm{d} s\right\} \prod_{r \in(0, t]}\left[\frac{j(r)}{\lambda}\right]^{\Delta N(r)}, \\
\operatorname{Re} Z(t)=\sum_{i=1}^{2} \int_{0}^{t}\left(m_{i}(s) \mathrm{d} B_{i}(s)-\frac{1}{2} m_{i}(s)^{2} \mathrm{~d} s\right) \\
m_{1}(t)=2 \operatorname{Im}\left(\overline{\alpha_{1}} \overline{h(t)} \xi(t)\right) \\
m_{2}(t)=2 \operatorname{Im}\left(\overline{\alpha_{2}} \xi(t)\right), \quad j|\beta|^{2}|\xi(t)|^{2} .
\end{gathered}
$$

Moreover, in Sec. IVA2 we find $L_{3}=0$ and in Sec. IVB $L_{4}(t)=0$ and $L_{i}, i \geq 5$, anti-selfadjoint (49). By the expression (11) for $m_{i}$, this gives $m_{i}(t)=0$ for $i=$ $3, \ldots, d$.

a. Girsanov transformation. As discussed in Sec. IIBOb, under the physical probability $\mathbb{P}_{\phi_{0}}^{T}$ the process $N(t)$ is a counting process of intensity $j(t)$ and $\left(W_{1}, W_{2}, B_{3}, \ldots, B_{d}\right)$ is a standard Wiener process and, in particular, its components are independent; the first two components are defined by Eq. (15).

b. Assumption on $f$ and $h$. We assume the phase diffusion model for the stimulating laser without feedback, so that the laser wave $f(t)$ is given by Eq. (35) and depends only on $B_{3}$. Similarly we assume the local oscillator $h(t)$ to be of the form (38) or (39), without feedback.

c. Stochastic independence. The Girsanov transformation of Sec. IV C 3a, the assumptions of Sec. IV C 3b, and the results expressed by Eqs. (65)- 60 and (73) have important consequences. First of all, under the physical probability, $\left(W_{1}, W_{2}\right)$ is independent of $\left(B_{3}, \ldots, B_{d}\right)$ and $\xi_{0}$, which is $\mathscr{F}_{0}$-measurable, and, so, $\left(W_{1}, W_{2}\right)$ is independent of $\left(\xi, h, f, m_{1}, m_{2}, j\right)$. Then, because $\left(B_{3}, \ldots, B_{d}\right)$ is a Wiener process below the reference probability and the physical probability, the law of $\left(f, h, U_{f}, U_{Y}, m_{1}, m_{2}, j\right)$ is the same below $\mathbb{Q}$ and $\mathbb{P}_{\phi_{0}}^{T}$. Let us stress that these properties are specific to the linear model constructed in this section, not to the general theory of Secs. II and III. 
d. The a priori state. From Eqs. (21), (22), and (72) and the properties discussed in Sec. IVC 3c we get that the a priori states are given by

$$
\eta(t)=\mathbb{E}_{\mathbb{P}_{\phi_{0}}^{T}}[|\psi(t)\rangle\langle\psi(t)|]=\mathbb{E}_{\mathbb{Q}}[|e(\xi(t))\rangle\langle e(\xi(t))|] .
$$

Note that $\eta(t)$ is a classical mixture of coherent states. Indeed, to keep the example simple, we introduced only interactions leaving invariant such a class of states.

e. The initial condition. In the next two sections we shall study the detection outputs under the physical probability $\mathbb{P}_{\phi_{0}}^{T}$. As we shall be interested only in the long-time behavior, no result will depend on the initial condition and from now on we take

$$
\xi_{0}=0 \text {. }
$$

\section{Direct detection}

The output current of direct detection is $J(t)$, given by Eq. (28), where $N$ is a counting process of stochastic intensity $j(t)(73 \mathrm{~b})$ :

$$
j(t)=\lambda|\beta|^{2}\|a \psi(t)\|^{2}=\lambda|\beta|^{2}|\xi(t)|^{2} .
$$

Let $\left(\mathscr{D}_{t}\right)$ be the natural filtration of the processes $f(\cdot)$ and $Y(\cdot)$ and set $\mathscr{D}:=\bigvee_{t \geq 0} \mathscr{D}_{t}$. The stochastic intensity $j(t)$ of the counting process $N$ is due only to the presence of $f$ and $Y$ in $\xi$. Therefore, conditionally on $\mathscr{D}, N$ is a time-inhomogeneous Poisson process. But, the law of the process $j$ is the same under the physical probability and under the reference probability; this gives the result that the characteristic functional of $N$ is

$$
\begin{aligned}
\Phi_{T}^{N}[k]:= & \mathbb{E}_{\mathbb{P}_{\phi_{0}}^{T}}\left[\exp \left\{\mathrm{i} \int_{0}^{T} k(t) \mathrm{d} N(t)\right\}\right] \\
& =\mathbb{E}_{\mathbb{Q}}\left[\exp \left\{\int_{0}^{T}\left(\mathrm{e}^{\mathrm{i} k(t)}-1\right) j(t) \mathrm{d} t\right\}\right] .
\end{aligned}
$$

Similarly, one can identify all the exclusive probability densities. By writing

$$
\begin{aligned}
\Phi_{T}^{N}[k]= & P_{T}(0)+\sum_{n=1}^{\infty} \int_{0}^{T} \mathrm{~d} t_{n} \int_{0}^{t_{n}} \mathrm{~d} t_{n-1} \\
& \cdots \int_{0}^{t_{2}} \mathrm{~d} t_{1} \exp \left\{\mathrm{i} \sum_{i=1}^{n} k\left(t_{i}\right)\right\} p_{T}\left(t_{n}, \ldots, t_{1}\right),
\end{aligned}
$$

we have that the probability of no counts up to time $T$ is

$$
P_{T}(0)=\mathbb{E}_{\mathbb{Q}}\left[\mathrm{e}^{-\int_{0}^{T} j(t) \mathrm{d} t}\right]
$$

and the probability density of a count around time $t_{1}, \ldots$, a count around time $t_{n}$ and no other count in between is

$$
p_{T}\left(t_{n}, \ldots, t_{1}\right)=\mathbb{E}_{\mathbb{Q}}\left[j\left(t_{n}\right) \cdots j\left(t_{1}\right) \mathrm{e}^{-\int_{0}^{T} j(t) \mathrm{d} t}\right] .
$$

From Eq. (76) one obtains also the expressions for all the multi-time correlation functions of the processes $N$ and $J$. In particular we get

$$
\begin{array}{r}
\mathbb{E}_{\mathbb{P}_{\phi_{0}}^{T}}\left[\int_{0}^{T} k(t) \mathrm{d} N(t)\right]=\int_{0}^{T} \mathrm{~d} t k(t) \mathbb{E}_{\mathbb{Q}}[j(t)] \\
\mathbb{E}_{\mathbb{P}_{\phi_{0}}^{T}}\left[\int_{0}^{T} k_{1}(t) \mathrm{d} N(t) \int_{0}^{T} k_{2}(s) \mathrm{d} N(s)\right] \\
=\int_{0}^{T} \mathrm{~d} t k_{1}(t) k_{2}(t) \mathbb{E}_{\mathbb{Q}}[j(t)] \\
+\int_{0}^{T} \mathrm{~d} t \int_{0}^{T} \mathrm{~d} s k_{1}(t) k_{2}(s) \mathbb{E}_{\mathbb{Q}}[j(t) j(s)] .
\end{array}
$$

\section{The mean counting intensity}

By taking the expressions (28) and (73b) for the output current $J$ and for the stochastic intensity $j$, we get

$$
\mathbb{E}_{\mathbb{P}_{\phi_{0}}^{t}}[J(t)]=\lambda|\beta|^{2} \int_{0}^{t} \mathrm{~d} r F_{J}(t-r) \mathbb{E}_{\mathbb{Q}}\left[|\xi(r)|^{2}\right] .
$$

As we show in Appendix B 1 a the mean of $|\xi(t)|^{2}$ has a limit $\Lambda$ for large times and, by taking the response function $F_{J}$ such that $\int_{0}^{+\infty} F_{J}(t) \mathrm{d} t=1$, we get

$$
\lim _{t \rightarrow+\infty} \mathbb{E}_{\mathbb{P}_{\phi_{0}}^{t}}[J(t)]=\lim _{t \rightarrow+\infty} \mathbb{E}_{\mathbb{Q}}[j(t)]=\lambda|\beta|^{2} \Lambda
$$

where

$$
\begin{gathered}
\Lambda=\Lambda_{f}+\sum_{j=5}^{d} \Lambda_{j}, \\
\Lambda_{f}=\frac{\left|\alpha_{2}\right|^{2}|g|^{2}\left(\gamma_{0}+\varepsilon\right)}{\gamma_{0}\left(\frac{\left(\gamma_{0}+\varepsilon\right)^{2}}{4}+(\Delta \nu)^{2}\right)}, \\
\Lambda_{j}=\frac{1}{2 \pi} \int_{-\infty}^{+\infty} \frac{\left|b_{j}+s_{j}(-\mathrm{i} x)\right|^{2}}{\left|\varkappa_{0}+\mathrm{i} x\right|^{2}} \mathrm{~d} x ;
\end{gathered}
$$

$s_{j}$ is the Laplace transform (53).

a. Incoherent light. In the case of incoherent light with the Lorentzian spectrum (57), described in Sec. IVB 2a, the contribution to $\Lambda$ is

$$
\Lambda_{5}=\frac{\left|g_{5}\right|^{2}\left(\gamma_{0}+\gamma_{5}\right)}{\gamma_{0} \gamma_{5}\left|\varkappa_{0}+\overline{\varkappa_{5}}\right|^{2}}
$$

note that it has same form as the contribution of $f$.

b. Squeezed reservoir. In the case of the Markovian squeezed reservoir of Sec. IV B 2b we get $s_{6}=s_{7}=0$ and

$$
\Lambda_{6}+\Lambda_{7}=n,
$$

where the parameter $n$ is defined in Eq. (58). Note that the squeezing parameter $m$ does not contribute to $\Lambda$. 


\section{The Mandel Q-parameter}

To compute the full statistics of the counts, say all the exclusive probability densities, is not easy. A simple, significant parameter related to a counting statistics is the Mandel $Q$-parameter defined, for $t_{0} \geq 0$ and $t>0$, by

$$
Q_{t_{0}}(t):=\frac{\operatorname{Var}_{\mathbb{P}_{\phi_{0}}^{t+t_{0}}}\left[N\left(t+t_{0}\right)-N\left(t_{0}\right)\right]}{\mathbb{E}_{\mathbb{P}_{\phi_{0}}^{t+t_{0}}}\left[N\left(t+t_{0}\right)-N\left(t_{0}\right)\right]}-1
$$

Because for a Poisson process this parameter is zero, in quantum optics it is usual to say that in the case of a positive $Q$ parameter one has super-Poissonian light and sub-Poissonian light in the other case. Sub-Poissonian light is considered an indication of non-classical effects. By Eqs. (77) and (78) we get

$$
Q_{t_{0}}(t)=\frac{\operatorname{Var}_{\mathbb{Q}}\left[\int_{t_{0}}^{t+t_{0}} j(s) \mathrm{d} s\right]}{\mathbb{E}_{\mathbb{Q}}\left[\int_{t_{0}}^{t+t_{0}} j(s) \mathrm{d} s\right]} \geq 0 .
$$

The emitted light is always super-Poissonian; indeed, it is well established that non-classical light can not be obtained in a linear system.

For large times we can define

$$
Q(t):=\lim _{t_{0} \rightarrow+\infty} Q_{t_{0}}(t)
$$

This parameter is studied in Appendix B1b; its general expression is given by Eq. (B2). We have that $Q(t)$ is non decreasing, $Q(0)=0$, and the limit $\lim _{t \rightarrow+\infty} Q(t)$ exists. The expression (B2) for $Q(t)$ shows that the contributions of $f$ and $Y$ interfere and the same holds for the various components of the process $Y$ : the situation for the Mandel $Q$-parameter is much more complex than for the mean intensity $\Lambda$, to which each channel contributes independently from the others, as seen in Sec. IV D 1

\section{E. Heterodyne and homodyne detection}

In terms of the new Wiener process, the hetero- or homodyne current $I(t)(\underline{30})$ can be written as

$$
\begin{array}{r}
I(t)=\int_{0}^{t} F_{I}(t-r)\left[m_{1}(r) \mathrm{d} r+\mathrm{d} W_{1}(r)\right], \\
m_{1}(t)=2 \operatorname{Re}\left(-\mathrm{i} \overline{\alpha_{1}} \overline{h(t)}\langle\psi(t) \mid a \psi(t)\rangle\right) \\
=2 \operatorname{Im}\left(\overline{\alpha_{1}} \overline{h(t)} \xi(t)\right) .
\end{array}
$$

As discussed in Sec. IV C 3 c in our linear model the processes $W_{1}$ and $m_{1}$ turn out to be stochastically independent under the physical probability, so that, modulo the detector response function $F_{I}$, we can interpret the output (88a) as signal, $m_{1}$, plus independent white noise, $\dot{W}_{1}$.

By using Eqs. (65)- 68 and (88) and the fact that $U_{Y}$ has zero mean and is independent of $h$, we get the expression for the mean output current

$$
\begin{aligned}
& \mathbb{E}_{\mathbb{P}_{\phi_{0}}^{t}}[I(t)]=-2 \operatorname{Re} \overline{\alpha_{1}} \alpha_{2} \int_{0}^{t} \mathrm{~d} s \int_{0}^{s} \mathrm{~d} r F_{I}(t-s) \\
& \times \mathrm{e}^{-\overline{\varkappa_{0}}(s-r)} \mathbb{E}_{\mathbb{Q}}[\overline{h(s)} f(r)] .
\end{aligned}
$$

Let us study now the spectrum of the hetero- or homodyne output, which can be obtained experimentally by a spectrum analyzer. The current $I(t)$ 88a is a classical stochastic process and again its spectrum is given by the classical definition [37]

$$
S_{I}(\mu)=\lim _{T \rightarrow+\infty} \frac{1}{T} \mathbb{E}_{\mathbb{P}_{\phi_{0}}^{T}}\left[\left.|| \int_{0}^{T} \mathrm{e}^{\mathrm{i} \mu t} I(t) \mathrm{d} t\right|^{2}\right] .
$$

In Appendix B2 we show that we have

$$
S_{I}(\mu)=\left|G_{I}(\mu)\right|^{2}\left[1+S_{m}(\mu)\right],
$$

where $G_{I}(\mu)$ is the Fourier transform (31) of the detector response function $F_{I}(t), 1$ is the constant contribution of the white noise $\dot{W}_{1}$ and

$$
S_{m}(\mu)=\lim _{T \rightarrow+\infty} \frac{1}{T} \mathbb{E}_{\mathbb{Q}}\left[\left.|| \int_{0}^{T} m_{1}(t) \mathrm{e}^{\mathrm{i} \mu t} \mathrm{~d} t\right|^{2}\right]
$$

From the expression (91) we see that the response function $F_{I}$ acts as a frequency filter $\left|G_{I}(\mu)\right|^{2}$; for instance, we can take any band-pass filter. Moreover, we have always $S_{I}(\mu) \geq\left|G_{I}(\mu)\right|^{2}$. This is due to the absence of correlations between $W_{1}$ and $m_{1}$ and it is interpreted as absence of squeezing in the quadratures of the emitted light, in agreement with the fact that linear systems cannot generate non-classical light. In contrast, a nonlinear system, such as a two-level atom, can generate squeezed fluorescent light and this can be treated by the formalism of SSE and continuous measurements [14, 32].

As discussed in Appendix B2, the spectrum $S_{m}(\mu)$ can be computed, and we obtain

$$
S_{m}(\mu)=S_{11}(\mu)+S_{12}(\mu)+S_{2}\left(\nu_{4}+\mu\right)+S_{2}\left(\nu_{4}-\mu\right),
$$

where the first two contributions are given by Eqs. (B3) and (B4) and

$$
S_{2}(\mu)=\int_{-\infty}^{+\infty} \mathrm{d} x \frac{2 \gamma_{4}\left|\alpha_{1}\right|^{2} \sum_{j=5}^{d}\left|b_{j}+s_{j}(-\mathrm{i} x)\right|^{2}}{\pi\left(\gamma_{4}^{2}+4(\mu-x)^{2}\right)\left|\varkappa_{0}+\mathrm{i} x\right|^{2}} ;
$$

here $\nu_{4}=\nu$ and $\gamma_{4}=\kappa$ for heterodyning or $\nu_{4}=\nu_{3}$ and $\gamma_{4}=\varepsilon$ for homodyning.

Let us stress that $S_{11}(\mu)+S_{12}(\mu)$ is the contribution to the fluorescent light of the laser wave $f(t)$, while $S_{2}\left(\nu_{4}+\right.$ 
$\mu)+S_{2}\left(\nu_{4}-\mu\right)$ is the contribution of the environment and of the incoherent light. Moreover, by comparing Eqs. (82) and (94), we obtain

$$
\frac{1}{4 \pi} \int_{-\infty}^{+\infty} S_{2}(\mu) \mathrm{d} \mu=\frac{\left|\alpha_{1}\right|^{2}}{2} \sum_{j=5}^{d} \Lambda_{j} .
$$

\section{Heterodyne spectrum}

In the case of heterodyning, the processes $f(35)$ and $h$ (39) are independent and this simplifies the computations of the fourth order moments involved in the expressions (B3) and (B4). In Appendix B2a we show that

$$
\begin{gathered}
S_{12}(\mu)=0, \\
S_{11}(\mu)=S_{1}(\nu+\mu)+S_{1}(\nu-\mu), \\
S_{1}(\nu)=\left|\alpha_{1}\right|^{2}\left|\alpha_{2}\right|^{2}|g|^{2}\left\{\frac{1}{\frac{(\kappa+\varepsilon)^{2}}{4}+\left(\nu_{3}-\nu\right)^{2}}\right. \\
\times\left[\frac{\kappa}{\frac{\left(\gamma_{0}+\varepsilon\right)^{2}}{4}+(\Delta \nu)^{2}}+\frac{\kappa \varepsilon}{\frac{\left(\gamma_{0}+\kappa\right)^{2}}{4}+\left(\nu-\nu_{0}\right)^{2}}\right] \\
+\frac{\left(\frac{\left(\gamma_{0}+\varepsilon\right)^{2}}{4}+(\Delta \nu)^{2}\right)\left(\frac{\left(\gamma_{0}+\kappa\right)^{2}}{4}+\left(\nu-\nu_{0}\right)^{2}\right)}{\left.\times\left[\frac{1}{\gamma_{0}}+\frac{\gamma_{0}+\kappa+\varepsilon}{\frac{(\kappa+\varepsilon)^{2}}{4}+\left(\nu_{3}-\nu\right)^{2}}\right]\right\} .}
\end{gathered}
$$

Recall that $\Delta \nu=\nu_{0}-\nu_{3}$ is the detuning.

By explicit computations, we find also that the mean current (89) does not contribute to the spectrum:

$$
\lim _{T \rightarrow+\infty} \frac{1}{T}\left|\int_{0}^{T} \mathrm{e}^{\mathrm{i} \mu t} \mathbb{E}_{\mathbb{P}_{\phi_{0}}^{T}}[I(t)] \mathrm{d} t\right|^{2}=0 .
$$

a. Intensity. Note that from Eqs. (81) and (98) we get

$$
\frac{1}{4 \pi} \int_{-\infty}^{+\infty} S_{1}(\mu) \mathrm{d} \mu=\frac{\left|\alpha_{1}\right|^{2}}{2} \Lambda_{f} .
$$

Equations (95) and (99) connect the intensities in direct detection and heterodyning, a general relation already encountered in other systems such as two-level atoms [14, 38].

b. Perfect local oscillator. To summarize, the heterodyne spectrum is given by Eqs. (91), (93), (94), (96), (97), and (98) with $\nu_{4}=\nu$ and $\gamma_{4}=\kappa$. These formulas are somewhat involved, as they contain contributions from various sources of noise: $\gamma_{0}$ is the natural with of the cavity mode, $\varepsilon$ is the width of the stimulating laser, and $\kappa$ is the width of the local oscillator. The situation becomes more transparent in the limit of a perfect local oscillator: $\kappa \downarrow 0$. By recalling that $\frac{\kappa}{2 \pi\left[(x+\nu)^{2}+\kappa^{2} / 4\right]} \rightarrow \delta(x+\nu)$, in this limit the heterodyne spectrum reduces to

$$
S_{m}(\mu)=\sum_{i=1}^{2}\left[S_{i}(\nu+\mu)+S_{i}(\nu-\mu)\right],
$$

$$
S_{1}(\nu \pm \mu)=\frac{\left|\alpha_{1}\right|^{2}\left|\alpha_{2}\right|^{2}}{\left(\nu \pm \mu-\nu_{0}\right)^{2}+\gamma_{0}^{2} / 4} S_{f}(\nu \pm \mu),
$$

$$
S_{2}(\nu \pm \mu)=\frac{\left|\alpha_{1}\right|^{2}}{\left(\nu \pm \mu-\nu_{0}\right)^{2}+\gamma_{0}^{2} / 4} S_{Y}(\nu \pm \mu) ;
$$

the spectra of the stimulating laser wave $f$ and of the incoherent noise $Y(t)$ are given by Eqs. (37) and (54). Let us recall that $\nu_{0}$ is the resonance frequency of our quantum system and $\nu$ is the frequency of the local oscillator in the detection apparatus, which can be adjusted by the experimenter. We can say that from the heterodyne output we can see part of the input spectra $S_{f}$ and $S_{Y}$ through a window of width $\gamma_{0}$ centered on $\nu_{0}$. In principle, in the case of a bad cavity with a big width $\gamma_{0}$, we can read the Markovian and non-Markovian character of the various contributions to the dynamics from the heterodyne spectrum.

\section{Homodyne spectrum}

The homodyne spectrum is given again by the general formulas (90), (91), and (92). Now, in the homodyne scheme, the local oscillator and the stimulating laser wave come from the same source, in order to maintain phase coherence during the detection process and to get a phase sensitive measurement procedure. However, this does not give coherence between the local oscillator and the various sources of noise described by the process $Y(t)$ (thermal reservoirs, incoherent light, etc.) and, indeed, the related contribution $S_{2}(\mu)$ to the homodyne spectrum is the same as in heterodyning and it is given by Eq. (94) with the substitutions $\nu_{4} \rightarrow \nu_{3}$ and $\gamma_{4} \rightarrow \varepsilon$.

The situation is different in the case of the components $S_{11}(\mu)$ (B3) and $S_{12}(\mu)$ (B4), which contain both the stimulating laser wave $f$ (35) and the local oscillator (38). To simplify the computations we consider only two limiting cases. The first case is when the optical paths of laser wave and local oscillator are much larger than the coherence length of the source; this is done by taking a large time delay $\Delta t$ and this washes out any phase sensitivity. The second case is when the two optical paths are perfectly balanced $(\Delta t=0)$ and the phase sensitivity is maximal. The general case is in between these two extremes.

a. The case $\Delta t \rightarrow \pm \infty$. By analyzing the expressions (B3) and (B4), one can see that in this limit $f$ and $h$ become stochastically independent and $S_{11}$ and 
$S_{12}$ become the same as in the heterodyne case with the substitutions $\nu \rightarrow \nu_{3}$ and $\kappa \rightarrow \varepsilon$, i.e. $S_{12}(\mu)=0$, $S_{11}(\mu)=S_{1}\left(\nu_{3}+\mu\right)+S_{1}\left(\nu_{3}-\mu\right)$ and

$$
\begin{array}{r}
S_{1}\left(\nu_{3} \pm \mu\right)=\frac{\varepsilon\left|\alpha_{1}\right|^{2}\left|\alpha_{2}\right|^{2}|g|^{2}}{\varepsilon^{2}+\mu^{2}}\left[\frac{1}{\frac{\left(\gamma_{0}+\varepsilon\right)^{2}}{4}+(\Delta \nu)^{2}}\right. \\
\left.+\frac{1}{\frac{\left(\gamma_{0}+\varepsilon\right)^{2}}{4}+(\Delta \nu \mp \mu)^{2}}\right]+\left[\frac{1}{\gamma_{0}}+\frac{\gamma_{0}+2 \varepsilon}{\varepsilon^{2}+\mu^{2}}\right] \\
\times \frac{\varepsilon^{2}\left|\alpha_{1}\right|^{2}\left|\alpha_{2}\right|^{2}|g|^{2}}{\left(\frac{\left(\gamma_{0}+\varepsilon\right)^{2}}{4}+(\Delta \nu)^{2}\right)\left(\frac{\left(\gamma_{0}+\varepsilon\right)^{2}}{4}+(\Delta \nu \mp \mu)^{2}\right)} .
\end{array}
$$

b. The case $\Delta t=0$. As shown in Appendix B2b, in this case we have

$$
\begin{gathered}
S_{11}(\mu)+S_{12}(\mu)=\frac{2\left|\alpha_{1}\right|^{2}\left|\alpha_{2}\right|^{2}|g|^{2}}{\frac{\left(\gamma_{0}+\varepsilon\right)^{2}}{4}+(\Delta \nu)^{2}}\left\{(\cos \zeta)^{2} 4 \pi \delta(\mu)\right. \\
+\frac{\varepsilon}{\gamma_{0}} \operatorname{Re}\left[\left(\frac{1}{\frac{\gamma_{0}+\varepsilon}{2}-\mathrm{i}(\Delta \nu-\mu)}+\frac{1}{\frac{\gamma_{0}+\varepsilon}{2}-\mathrm{i}(\Delta \nu+\mu)}\right)\right. \\
\left.\left.\times\left(1-\frac{\gamma_{0} \mathrm{e}^{2 \mathrm{i} \zeta}}{\gamma_{0}+2 \varepsilon-2 \mathrm{i} \Delta \nu}\right)\right]\right\}, \quad(102 \\
\zeta:=\arg \left(\frac{\alpha_{1} \overline{\alpha_{2}}}{\frac{\gamma_{0}+\varepsilon}{2}-\mathrm{i} \Delta \nu}\right)+\theta .
\end{gathered}
$$

Let us stress that $\Delta t=0$ means that the two optical paths are perfectly balanced and homodyning allows for perfect interference, which generates a $\delta$ spike at zero frequency. By varying $|\Delta t|$ from zero to infinity, one goes from (102) to (101) and $S_{12}(\mu)=0$.

From Eq. (102) we see that, by adjusting the phase $\zeta$, we can change the relative intensity of the $\delta$ spike and the regular part. To simplify, let us consider only two extreme cases. We take always a vanishing detuning, which implies, in particular, $\zeta=\arg \left(\alpha_{1} \overline{\alpha_{2}}\right)+\theta$. First we define

$$
l(\mu):=\frac{16\left|\alpha_{1}\right|^{2}\left|\alpha_{2}\right|^{2}|g|^{2}}{\gamma_{0}\left[\left(\frac{\gamma_{0}+\varepsilon}{2}\right)^{2}+\mu^{2}\right]} .
$$

Then, for $\Delta \nu=0$ and $\zeta= \pm \pi / 2$, we have

$$
S_{11}(\mu)+S_{12}(\mu)=\frac{\varepsilon}{\gamma_{0}+2 \varepsilon} l(\mu) .
$$

In the other extreme case, $\Delta \nu=0$ and $\zeta=0$ or $\pi$, we have

$$
S_{11}(\mu)+S_{12}(\mu)=\frac{\varepsilon^{2} l(\mu)}{\left(\gamma_{0}+\varepsilon\right)\left(\gamma_{0}+2 \varepsilon\right)}+\frac{\pi}{2} \gamma_{0} l(0) \delta(\mu) .
$$

Let us stress that this phase sensitivity is the characterizing feature of homodyne detection.

\section{CONCLUSIONS}

In this article we have presented a SSE in which the involved operators are allowed to be random and to depend on the past; moreover, the driving noises can be colored diffusive processes and general counting processes, not only white noise and Poisson processes. This modification introduces memory without violating the complete positivity of the dynamics for the reduced state (the $a$ priori state or mean state). In this way we have an unravelling of a completely positive dynamics with memory. The main difference with respect the Markovian case is that now a random Liouville operator appears and this precludes having a simple closed equation for the mean dynamics.

By constructing positive operator-valued measures and instruments, we have also shown that our proposal is compatible with an interpretation in terms of continuous monitoring of the system; the axiomatic structure of quantum mechanics is respected. The key point in the construction is that the starting point is the linear $S S E$ and that its structure is such that the square norm of the solution is a martingale.

On physical grounds, this theory allows various memory effects to be introduced in a consistent way. The most important one is the possibility of introducing measurement-based feedback, with delay, a fact that opens the way to a general treatment of quantum closedloop control. Memory and feedback can be introduced also in the detection part (say in the local oscillator of a hetero- or homodyne detector) and this allows for a consistent treatment of adaptive measurements.

In order to understand the possibilities of the theory, we have introduced the simplest quantum system, a harmonic oscillator with a dynamics leaving invariant the coherent states. The possibility of using random terms in the Liouville operator allows the introduction various memory effects, such as colored environments, producing non-white thermal-like effects with any spectral density. Moreover, the stimulating light can be a laser with imperfections (we have taken the phase diffusion model of a laser wave) and/or incoherent light (thermal light, for instance).

As possible continuous monitoring, we first studied direct detection and showed how the detected intensity and the Mandel $Q$ parameter depend on the characteristics of the input (light and thermal effects).

Then we studied the spectrum of the hetero- or homodyne current. Here, the imperfections in the local oscillator can also be consistently introduced in the theory. The two spectra are affected by all the noises and characteristics of the dynamics and have somewhat complicated expressions; however, two features appear. The heterodyne spectrum reproduces a part of the spectra of the thermal noises and of the stimulating light and, so, in principle, it allows us to see directly the sources of nonMarkovian effects. The homodyne spectrum turns out to be phase sensitive, as is known; by controlling the initial 
phase it is possible to change the field quadrature that is detected. The possibility of introducing a coherence length in the stimulating light and in the local oscillator allows a study of the influence of imperfections on homodyning; one sees that, as soon the apparatus is not perfectly balanced, the homodyne spectrum becomes the same as the heterodyne spectrum.

\section{Appendix A: Some autocorrelation functions}

\section{The stimulating laser}

Let us consider the process (35) modeling the stimulating laser light. We can say that $f$ is a log-normal process, i.e., the exponential of a (complex) Gaussian process. In stochastic calculus it is well known that $\exp \left\{\mathrm{i} \sqrt{\varepsilon} B_{3}(t)+\varepsilon t / 2\right\}$ is a mean-1 martingale, which gives the mean function

$$
\mathbb{E}_{\mathbb{Q}}[f(t)]=g \mathrm{e}^{-\mathrm{i} \nu_{3} t-\varepsilon t / 2}
$$

and the autocorrelation functions

$$
\begin{gathered}
\mathbb{E}_{\mathbb{Q}}[f(r) \overline{f(s)}]=|g|^{2} \mathrm{e}^{\mathrm{i} \nu_{3}(s-r)-\varepsilon|s-r| / 2}, \\
\mathbb{E}_{\mathbb{Q}}[f(r) f(s)]=g^{2} \mathrm{e}^{-\left(\mathrm{i} \nu_{3}+\varepsilon / 2\right)(s+r)-\varepsilon(s \wedge r)},
\end{gathered}
$$

where $a \wedge b$ is the minimum between $a$ and $b$. In the proof of Eqs. (A2) and A3 one has to use (A1) and the independence of the increments of the Wiener process.

Let us stress that from (A2) we get immediately the spectrum (37), while from (A1) and (A3) we get

$$
\begin{aligned}
& \lim _{T \rightarrow+\infty} \frac{1}{T}\left|\int_{0}^{T} \mathrm{e}^{\mathrm{i} \mu t} \mathbb{E}_{\mathbb{Q}}[f(t)] \mathrm{d} t\right|^{2}=0, \\
& \lim _{T \rightarrow+\infty} \frac{1}{T} \mathbb{E}_{\mathbb{Q}}\left[\left(\int_{0}^{T} \mathrm{e}^{\mathrm{i} \mu t} f(t) \mathrm{d} t\right)^{2}\right]=0 .
\end{aligned}
$$

We shall need also the autocorrelation function of the related process $U_{f}(t)$ for large times. From (66), (A2), and (A3) we obtain

$$
\begin{aligned}
\lim _{t_{0} \rightarrow+\infty} \mathbb{E}_{\mathbb{Q}}\left[U_{f}\left(t+t_{0}\right) U_{f}\left(s+t_{0}\right)\right]=0, \\
\lim _{t_{0} \rightarrow+\infty} \mathbb{E}_{\mathbb{Q}}\left[U_{f}\left(t+t_{0}\right) \overline{U_{f}\left(s+t_{0}\right)}\right] \\
=\frac{\left|\alpha_{2}\right|^{2}|g|^{2}}{\gamma_{0}}\left\{\frac{\mathrm{e}^{-\overline{\varkappa_{0}}(t-s)}}{\overline{\varkappa_{0}}+\varkappa_{3}}+\frac{\mathrm{e}^{-\overline{\varkappa_{3}}(t-s)}}{\varkappa_{0}+\overline{\varkappa_{3}}}\right. \\
\left.+\frac{\mathrm{e}^{-\overline{\varkappa_{3}}(t-s)}-\mathrm{e}^{-\overline{\varkappa_{0}}(t-s)}}{\overline{\varkappa_{0}}-\overline{\varkappa_{3}}}\right\}, \quad t \geq s,
\end{aligned}
$$

where $\varkappa_{3}=\frac{\varepsilon}{2}-\mathrm{i} \nu_{3}$.

\section{Autocorrelation functions of the process $Y$}

Let us consider the Gaussian process $Y$ defined by Eqs. (43) and (44); we can write

$$
\begin{array}{rl}
\int_{0}^{T} \mathrm{e}^{\mathrm{i} \mu t} \mathrm{~d} Y(t)=\sum_{j=5}^{d} \int_{0}^{T} & \mathrm{~d} B_{j}(t) \mathrm{e}^{\mathrm{i} \mu t} \\
& \times\left(b_{j}+\int_{0}^{T-t} \mathrm{~d} s \mathrm{e}^{\mathrm{i} \mu s} c_{j}(s)\right) .
\end{array}
$$

Then, by using the Itô isometry we get

$$
\begin{array}{r}
\frac{1}{T} \mathbb{E}_{\mathbb{Q}}\left[\left|\int_{0}^{T} \mathrm{e}^{\mathrm{i} \mu t} \mathrm{~d} Y(t)\right|^{2}\right] \\
=\frac{1}{T} \sum_{j=5}^{d} \int_{0}^{T} \mathrm{~d} t\left|b_{j}+\int_{0}^{t} \mathrm{~d} s c_{j}(s) \mathrm{e}^{\mathrm{i} \mu s}\right|^{2}
\end{array}
$$

From the limit $T \rightarrow+\infty$ we get immediately the Laplace transform (53) inside the square modulus and (54) is proved.

The expression (43) of the process $Y$ contains the function $c_{j}(t)$ and its spectrum involves the Laplace transform $s_{j}(z)$ (53) of $c_{j}$. In the following, we shall need also the Fourier inversion formula:

$$
c_{j}(t)=\frac{1}{2 \pi} \int_{-\infty}^{+\infty} \mathrm{e}^{-\mathrm{i} \mu t} s_{j}(-\mathrm{i} \mu) \mathrm{d} \mu, \quad t>0 .
$$

Similarly to (53) and (A6), for the function $g_{j}(t)$ (68) we have

$$
\begin{gathered}
\int_{0}^{+\infty} \mathrm{e}^{-z t} g_{j}(t) \mathrm{d} t=\frac{b_{j}+s_{j}(z)}{\overline{\varkappa_{0}}+z}, \quad \operatorname{Re} z \geq 0, \\
g_{j}(t)=\frac{1}{2 \pi} \int_{-\infty}^{+\infty} \mathrm{e}^{-\mathrm{i} x t} \frac{b_{j}+s_{j}(-\mathrm{i} x)}{\overline{\varkappa_{0}}-\mathrm{i} x} \mathrm{~d} x, \quad t>0 .
\end{gathered}
$$

For the related process $U_{Y}(t)$, from Eqs. (67), (A7), and (A8) we obtain, with $t \geq s$,

$$
\begin{aligned}
& \lim _{t_{0} \rightarrow+\infty} \mathbb{E}_{\mathbb{Q}}\left[U_{Y}\left(t+t_{0}\right) \overline{U_{Y}\left(s+t_{0}\right)}\right] \\
& =\frac{1}{2 \pi} \int_{-\infty}^{+\infty} \mathrm{e}^{-\mathrm{i} x(t-s)} \sum_{j=5}^{d} \frac{\left|b_{j}+s_{j}(-\mathrm{i} x)\right|^{2}}{\left|\varkappa_{0}+\mathrm{i} x\right|^{2}} \mathrm{~d} x \\
& \lim _{t_{0} \rightarrow+\infty} \mathbb{E}_{\mathbb{Q}}\left[U_{Y}\left(t+t_{0}\right) U_{Y}\left(s+t_{0}\right)\right]=\frac{1}{2 \pi} \int_{-\infty}^{+\infty} \mathrm{d} x \\
& \quad \times \mathrm{e}^{-\mathrm{i} x(t-s)} \sum_{j=5}^{d} \frac{\left[b_{j}+s_{j}(-\mathrm{i} x)\right]\left[b_{j}+s_{j}(\mathrm{i} x)\right]}{\bar{\varkappa}^{2}+x^{2}}
\end{aligned}
$$




\section{The local oscillator in the heterodyne scheme}

For the moments of the local oscillator in the heterodyne measurement scheme (39) we have formulas analogous to those of Appendix A1.

$$
\begin{gathered}
\mathbb{E}_{\mathbb{Q}}[h(t)]=\mathrm{e}^{\mathrm{i} \vartheta} \mathrm{e}^{-\mathrm{i} \nu t-\kappa t / 2}, \\
\mathbb{E}_{\mathbb{Q}}[\overline{h(t)} h(s)]=\mathrm{e}^{\mathrm{i} \nu(t-s)-\kappa|t-s| / 2}, \\
\mathbb{E}_{\mathbb{Q}}\left[\overline{h(t)} \frac{\overline{h(s)}]=\mathrm{e}^{-2 \mathrm{i} \vartheta} \mathrm{e}^{(\mathrm{i} \nu-\kappa / 2)(t+s)-\kappa(t \wedge s)} .}{}\right.
\end{gathered}
$$

\section{The local oscillator in the homodyne scheme}

In the homodyne measurement scheme the local oscillator $h$ is proportional to the shifted stimulating laser $f$ and it is given by Eq. (38), i.e.,

$$
h(t)=\frac{g \mathrm{e}^{\mathrm{i} \theta}}{|g|} \mathrm{e}^{-\mathrm{i} \nu_{3}(t-\Delta t)+\mathrm{i} \sqrt{\varepsilon} B_{3}(t-\Delta t)} .
$$

As before we get

$$
\begin{gathered}
\mathbb{E}_{\mathbb{Q}}[h(t)]=\frac{g \mathrm{e}^{\mathrm{i} \theta}}{|g|} \mathrm{e}^{-\mathrm{i} \nu_{3}(t-\Delta t)-\varepsilon|t-\Delta t| / 2}, \\
\mathbb{E}_{\mathbb{Q}}[\overline{h(t)} h(s)]=\mathrm{e}^{\mathrm{i} \nu_{3}(t-s)-\varepsilon|t-s| / 2}, \\
\mathbb{E}_{\mathbb{Q}}[\overline{h(t)} \overline{h(s)}]=\left(\frac{g \mathrm{e}^{-\mathrm{i} \theta}}{|g|}\right)^{2} \mathrm{e}^{\left(\mathrm{i} \nu_{3}-\frac{\varepsilon}{2}\right)(t+s-2 \Delta t)-\varepsilon(t \wedge s-\Delta t)} .
\end{gathered}
$$

In the homodyne case we need also the two fourth order moments

$$
\begin{gathered}
\mathbb{E}_{\mathbb{Q}}\left[f\left(r_{1}\right) \overline{f\left(r_{2}\right)} \overline{h(t)} h(s)\right]=|g|^{2} \exp \left\{\mathrm{i} \nu_{3}\left(t-r_{1}-s+r_{2}\right)-\frac{\varepsilon}{2} \delta\left(r_{1}, t-\Delta t, s-\Delta t, r_{2}\right)\right\}, \\
\mathbb{E}_{\mathbb{Q}}\left[f\left(r_{1}\right) f\left(r_{2}\right) \overline{h(t)} \overline{h(s)}\right]=|g|^{2} \exp \left\{\mathrm{i} \nu_{3}\left(t-r_{1}+s-r_{2}-2 \Delta t\right)-\frac{\varepsilon}{2} \delta\left(r_{1}, t-\Delta t, r_{2}, s-\Delta t\right)\right\},
\end{gathered}
$$

where

$$
\exp \left\{-\frac{\varepsilon}{2} \delta\left(t_{1}, t_{2}, t_{3}, t_{4}\right)\right\}=\mathbb{E}_{\mathbb{Q}}\left[\exp \left\{\mathrm{i} \sqrt{\varepsilon}\left(B_{3}\left(t_{1}\right)-B_{3}\left(t_{2}\right)+B_{3}\left(t_{3}\right)-B_{3}\left(t_{4}\right)\right)\right\}\right] .
$$

By some long, but straightforward computations we get

$$
\delta\left(t_{1}, t_{2}, t_{3}, t_{4}\right)= \begin{cases}\left|t_{4}-t_{3}\right|+\left|t_{2}-t_{1}\right|, & \text { for } t_{1} \vee t_{2}<t_{3} \wedge t_{4} \text { or } t_{3} \vee t_{4}<t_{1} \wedge t_{2}, \\ \left|t_{4}-t_{1}\right|+\left|t_{3}-t_{2}\right|, & \text { for } t_{1} \vee t_{4}<t_{2} \wedge t_{3} \text { or } t_{2} \vee t_{3}<t_{1} \wedge t_{4}, \\ \left|t_{4}-t_{1}\right|+3\left|t_{3}-t_{2}\right|, & \text { for } t_{4}<t_{2}<t_{3}<t_{1} \text { or } t_{1}<t_{3}<t_{2}<t_{4}, \\ \left|t_{4}-t_{3}\right|+3\left|t_{2}-t_{1}\right|, & \text { for } t_{4}<t_{2}<t_{1}<t_{3} \text { or } t_{3}<t_{1}<t_{2}<t_{4}, \\ \left|t_{2}-t_{3}\right|+3\left|t_{4}-t_{1}\right|, & \text { for } t_{2}<t_{4}<t_{1}<t_{3} \text { or } t_{3}<t_{1}<t_{4}<t_{2}, \\ \left|t_{2}-t_{1}\right|+3\left|t_{3}-t_{4}\right|, & \text { for } t_{2}<t_{4}<t_{3}<t_{1} \text { or } t_{1}<t_{3}<t_{4}<t_{2} .\end{cases}
$$

\section{Appendix B: Detection}

is Gaussian with mean zero. So we have

\section{Direct detection}

a. The mean counting intensity

The expression of $\xi(t)$ is given by (65); moreover, the processes $U_{f}$ (66) and $U_{Y}$ (67) are independent and $U_{Y}$

$$
\mathbb{E}_{\mathbb{Q}}\left[|\xi(t)|^{2}\right]=\mathbb{E}_{\mathbb{Q}}\left[\left|U_{f}(t)\right|^{2}\right]+\mathbb{E}_{\mathbb{Q}}\left[\left|U_{Y}(t)\right|^{2}\right]
$$


From and and we get

$$
\begin{aligned}
\mathbb{E}_{\mathbb{Q}}\left[|\xi(t)|^{2}\right]= & \frac{\left|\alpha_{2}\right|^{2}|g|^{2}\left(\gamma_{0}+\varepsilon\right)}{\gamma_{0}\left(\frac{\left(\gamma_{0}+\varepsilon\right)^{2}}{4}+(\Delta \nu)^{2}\right)} \\
& +\frac{1}{2 \pi} \sum_{j=5}^{d} \int_{-\infty}^{+\infty} \frac{\left|b_{j}+s_{j}(-\mathrm{i} x)\right|^{2}}{\left|\varkappa_{0}+\mathrm{i} x\right|^{2}} \mathrm{~d} x .
\end{aligned}
$$

From Eq. (B1) we obtain the expression for $\Lambda$ (80).

\section{b. The Mandel parameter}

By using Eqs. (86), (87), (65), and (A4) and by recalling that the process $U_{Y}$ is Gaussian with zero mean and $U_{Y}(s)$ and $U_{f}(r)$ are independent, we get

$$
\begin{aligned}
& Q(t)=\frac{2 \lambda|\beta|^{2} \gamma_{0}}{\Lambda t} \\
& \times \lim _{t_{0}} \rightarrow+\infty \int_{t_{0}}^{t+t_{0}} \mathrm{~d} s \int_{t_{0}}^{s} \mathrm{~d} r\left\{\left|\mathbb{E}_{\mathbb{Q}}\left[\overline{U_{Y}(s)} U_{Y}(r)\right]\right|^{2}\right. \\
&+\left|\mathbb{E}_{\mathbb{Q}}\left[U_{Y}(s) U_{Y}(r)\right]\right|^{2}+\operatorname{Cov}_{\mathbb{Q}}\left[\left|U_{f}(s)\right|^{2},\left|U_{f}(r)\right|^{2}\right] \\
&\left.\quad+2 \operatorname{Re} \mathbb{E}_{\mathbb{Q}}\left[U_{f}(s) \overline{U_{f}(r)}\right] \mathbb{E}_{\mathbb{Q}}\left[\overline{U_{Y}(s)} U_{Y}(r)\right]\right\} .
\end{aligned}
$$

We see that $Q(0)=0$; moreover, for large times the integrand depends only on the difference $s-r$ and one can check that $\dot{Q}(t) \geq 0$. So $Q(t)$ is non decreasing and

$$
\begin{gathered}
Q(t)=\frac{2 \lambda|\beta|^{2} \gamma_{0}}{\Lambda} \int_{0}^{t} \mathrm{~d} s\left(1-\frac{s}{t}\right) \\
\times \lim _{t_{0} \rightarrow+\infty}\left\{\left|\mathbb{E}_{\mathbb{Q}}\left[\overline{U_{Y}\left(t_{0}+s\right)} U_{Y}\left(t_{0}\right)\right]\right|^{2}\right. \\
\quad+\left|\mathbb{E}_{\mathbb{Q}}\left[U_{Y}\left(t_{0}+s\right) U_{Y}\left(t_{0}\right)\right]\right|^{2} \\
+2 \operatorname{Re} \mathbb{E}_{\mathbb{Q}}\left[U_{f}\left(t_{0}+s\right) \overline{U_{f}\left(t_{0}\right)}\right] \mathbb{E}_{\mathbb{Q}}\left[\overline{U_{Y}\left(t_{0}+s\right)} U_{Y}\left(t_{0}\right)\right] \\
\left.\quad+\operatorname{Cov}_{\mathbb{Q}}\left[\left|U_{f}\left(t_{0}+s\right)\right|^{2},\left|U_{f}\left(t_{0}\right)\right|^{2}\right]\right\} . \quad(\mathrm{B} 2)
\end{gathered}
$$

By using (A5), A9), and (A10) and the analogs of (A18) and (A19), one sees that $\lim _{t \rightarrow+\infty} Q(t)$ exists and it would be possible to compute $Q(t)$, but its general expression is very involved and not particularly instructive.

\section{Homo- and heterodyne spectra}

By (88a) and the independence of $W_{1}$ and $m_{1}$ under the physical probability $\mathbb{P}_{\phi_{0}}^{T}$, we get, by some changes of integration variables,

$$
\begin{aligned}
\frac{1}{T} \mathbb{E}_{\mathbb{P}_{\phi_{0}}}\left[\left|\int_{0}^{T} \mathrm{e}^{\mathrm{i} \mu t} I(t) \mathrm{d} t\right|^{2}\right] & =\frac{1}{T} \int_{0}^{T} \mathrm{~d} s\left|\int_{0}^{s} \mathrm{~d} t \mathrm{e}^{\mathrm{i} \mu t} F(t)\right|^{2} \\
+\frac{1}{T} \mathbb{E} & {\left[\left|\int_{0}^{T} \mathrm{~d} t \mathrm{e}^{\mathrm{i} \mu t} m_{1}(t) \int_{0}^{T-t} \mathrm{~d} s \mathrm{e}^{\mathrm{i} \mu s} F(s)\right|^{2}\right] . }
\end{aligned}
$$

Then, in the limit $T \rightarrow+\infty$, we obtain Eqs. (91) and (92).

To compute the spectrum $S_{m}(\mu)$ (92) we have to take the expression (73a) of $m_{1}$ and to use Eqs. (65)- (68). By recalling that $U_{Y}$ is Gaussian, with zero mean and independent of $h$ and $f$, by some computations we arrive at the expression $S_{m}(\mu)=S_{11}(\mu)+S_{12}(\mu)+\tilde{S}_{21}(\mu)+$ $\tilde{S}_{22}(\mu)$ with

$$
\begin{gathered}
S_{11}(\mu)=\lim _{T \rightarrow+\infty} \frac{4\left|\alpha_{1}\right|^{2}\left|\alpha_{2}\right|^{2}}{T} \operatorname{Re} \int_{0}^{T} \mathrm{~d} t \int_{0}^{t} \mathrm{~d} s \cos (\mu(t-s)) \mathrm{e}^{-\overline{\varkappa_{0}} t-\varkappa_{0} s}\left\{\int_{0}^{s} \mathrm{~d} r_{1} \int_{0}^{r_{1}} \mathrm{~d} r_{2} \mathrm{e}^{\overline{\varkappa_{0}} r_{2}+\varkappa_{0} r_{1}}\right. \\
\left.\times \mathbb{E}_{\mathbb{Q}}\left[\overline{f\left(r_{1}\right)} f\left(r_{2}\right) \overline{h(t)} h(s)\right]+\left(\int_{s}^{t} \mathrm{~d} r_{1} \int_{0}^{s} \mathrm{~d} r_{2}+\int_{0}^{s} \mathrm{~d} r_{1} \int_{0}^{r_{1}} \mathrm{~d} r_{2}\right) \mathrm{e}^{\overline{\varkappa_{0}} r_{1}+\varkappa_{0} r_{2}} \mathbb{E}_{\mathbb{Q}}\left[f\left(r_{1}\right) \overline{f\left(r_{2}\right)} \overline{h(t)} h(s)\right]\right\}, \\
S_{12}(\mu)=\lim _{T \rightarrow+\infty} \operatorname{Re} \frac{4{\overline{\alpha_{1}}}^{2} \alpha_{2}{ }^{2}}{T} \int_{0}^{T} \mathrm{~d} t \int_{0}^{t} \mathrm{~d} s \cos (\mu(t-s))\left(\int_{s}^{t} \mathrm{~d} r_{1} \int_{0}^{s} \mathrm{~d} r_{2}\right. \\
\left.+2 \int_{0}^{s} \mathrm{~d} r_{1} \int_{0}^{r_{1}} \mathrm{~d} r_{2}\right) \mathrm{e}^{-\overline{\varkappa_{0}}\left(t+s-r_{1}-r_{2}\right)} \mathbb{E}_{\mathbb{Q}}\left[f\left(r_{1}\right) f\left(r_{2}\right) \overline{h(t)} \overline{h(s)}\right],
\end{gathered}
$$




$$
\begin{gathered}
\tilde{S}_{21}(\mu)=\lim _{T \rightarrow+\infty} \frac{4\left|\alpha_{1}\right|^{2}}{T} \sum_{j=5}^{d} \operatorname{Re} \int_{0}^{T} \mathrm{~d} t \int_{0}^{t} \mathrm{~d} s \int_{0}^{s} \mathrm{~d} r \cos (\mu(t-s)) g_{j}(t-r) \overline{g_{j}(s-r)} \mathbb{E}_{\mathbb{Q}}[\overline{h(t)} h(s)], \\
\tilde{S}_{22}(\mu)=\lim _{T \rightarrow+\infty} \operatorname{Re} \frac{4 \bar{\alpha}_{1}^{2}}{T} \sum_{j=5}^{d} \int_{0}^{T} \mathrm{~d} t \int_{0}^{t} \mathrm{~d} s \int_{0}^{s} \mathrm{~d} r \cos (\mu(t-s)) g_{j}(t-r) g_{j}(s-r) \mathbb{E}_{\mathbb{Q}}[\overline{h(t)} \overline{h(s)}] .
\end{gathered}
$$

Let us consider now both heterodyning and homodyning; the moments (A12) and (A16) can be written in a unified way as

$$
\mathbb{E}_{\mathbb{Q}}[\overline{h(t)} h(s)]=\mathrm{e}^{-\varkappa_{4}(t-s)}, \quad t \geq s,
$$

with $\varkappa_{4}=\frac{\kappa}{2}-\mathrm{i} \nu$ for heterodyning, $\varkappa_{4}=\frac{\varepsilon}{2}-\mathrm{i} \nu_{3}$ for homodyning. Then, from (B5) we obtain

$$
\tilde{S}_{21}(\mu)=4\left|\alpha_{1}\right|^{2} \sum_{j=5}^{d} \operatorname{Re} \int_{0}^{+\infty} \mathrm{d} t \int_{0}^{t} \mathrm{~d} s \mathrm{e}^{-\varkappa_{4}(t-s)} \cos (\mu(t-s)) g_{j}(t) \overline{g_{j}(s)} .
$$

By inserting (A8) into (B7) and, then, by using (A7) for $\overline{g_{j}(s)}$, we get $\tilde{S}_{21}(\mu)=S_{2}\left(\nu_{4}+\mu\right)+S_{2}\left(\nu_{4}-\mu\right)$ with $S_{2}(\mu)$ given by (94).

By similar computations, from (B6) we find that $\tilde{S}_{22}(\mu)$ vanishes for both the choices (38) and (39) for the local oscillator $h(t)$; this ends the proof of (93) and (94).

\section{a. Heterodyne detection}

By using (A2) and (A12) inside (B3), we get (97) with

$$
\begin{aligned}
& S_{1}(\nu)= \lim _{T \rightarrow+\infty} \frac{2\left|\alpha_{1}\right|^{2}\left|\alpha_{2}\right|^{2}|g|^{2}}{T} \operatorname{Re} \int_{0}^{T} \mathrm{~d} t \int_{0}^{t} \mathrm{~d} s \mathrm{e}^{\left(\mathrm{i} \nu-\frac{\kappa}{2}\right)(t-s)}\left\{\left(\int_{s}^{t} \mathrm{~d} r_{1} \int_{0}^{s} \mathrm{~d} r_{2}+\int_{0}^{s} \mathrm{~d} r_{1} \int_{0}^{r_{1}} \mathrm{~d} r_{2}\right)\right. \\
&\left.\times \mathrm{e}^{-\overline{\varkappa_{0}}\left(t-r_{1}\right)-\varkappa_{0}\left(s-r_{2}\right)+\mathrm{i} \nu_{3}\left(r_{2}-r_{1}\right)-\frac{\varepsilon}{2}\left(r_{1}-r_{2}\right)}+\int_{0}^{s} \mathrm{~d} r_{1} \int_{0}^{r_{1}} \mathrm{~d} r_{2} \mathrm{e}^{-\overline{\varkappa_{0}}\left(t-r_{2}\right)-\varkappa_{0}\left(s-r_{1}\right)+\mathrm{i} \nu_{3}\left(r_{1}-r_{2}\right)-\frac{\varepsilon}{2}\left(r_{1}-r_{2}\right)}\right\} \\
& \quad=\operatorname{Re} \frac{2\left|\alpha_{1}\right|^{2}\left|\alpha_{2}\right|^{2}|g|^{2}}{\overline{\varkappa_{0}}+\frac{\kappa}{2}-\mathrm{i} \nu}\left\{\frac{1}{\varkappa_{0}+\frac{\varepsilon}{2}+\mathrm{i} \nu_{3}}\left[\frac{1}{\frac{\kappa+\varepsilon}{2}+\mathrm{i}\left(\nu_{3}-\nu\right)}+\frac{1}{\gamma_{0}}\right]+\frac{1}{\left(\overline{\varkappa_{0}}+\frac{\varepsilon}{2}-\mathrm{i} \nu_{3}\right) \gamma_{0}}\right\} .
\end{aligned}
$$

By explicit computations, from (B8) we get (98). Similarly, by (B4), (A33), and (A13) we obtain $S_{12}(\mu)=0$.

\section{b. Homodyning, $\Delta t=0$}

By inserting the expression (A18) into (B3) and (A19) into (B4), we obtain

$$
\begin{gathered}
S_{11}(\mu)=\frac{\left|\alpha_{1}\right|^{2}\left|\alpha_{2}\right|^{2}|g|^{2}}{\frac{\left(\gamma_{0}+\varepsilon\right)^{2}}{4}+(\Delta \nu)^{2}}\left\{4 \pi \delta(\mu)+\frac{\varepsilon\left(\gamma_{0}+\varepsilon\right)}{\gamma_{0}}\left[\frac{1}{\frac{\left(\gamma_{0}+\varepsilon\right)^{2}}{4}+(\mu+\Delta \nu)^{2}}+\frac{1}{\frac{\left(\gamma_{0}+\varepsilon\right)^{2}}{4}+(\mu-\Delta \nu)^{2}}\right]\right\}, \\
S_{12}(\mu)=\operatorname{Re} \frac{|g|^{2} \mathrm{e}^{2 \mathrm{i} \theta} \alpha_{1}^{2} \bar{\alpha}^{2}}{\left(\varkappa_{0}+\mathrm{i} \nu_{3}+\frac{\varepsilon}{2}\right)^{2}}\left\{4 \pi \delta(\mu)-\frac{\varepsilon}{\varkappa_{0}+\mathrm{i} \nu_{3}+\varepsilon}\left[\frac{1}{\varkappa_{0}+\mathrm{i}\left(\nu_{3}+\mu\right)+\frac{\varepsilon}{2}}+\frac{1}{\varkappa_{0}+\mathrm{i}\left(\nu_{3}-\mu\right)+\frac{\varepsilon}{2}}\right]\right\} .
\end{gathered}
$$

In doing the computations we have used the well known approximations of the Dirac $\delta \frac{\sin \mu T}{\pi \mu} \rightarrow \delta(\mu)$ and sions (B9) and (B10) we get (102). $\frac{2(\sin \mu T / 2)^{2}}{\pi T \mu^{2}}=\frac{1-\cos \mu T}{\pi T \mu^{2}} \rightarrow \delta(\mu)$. By adding the expres-

[1] H.-P. Breuer and F. Petruccione, The Theory of Open Quantum Systems (Oxford University Press, Oxford,

2002). 
[2] H.J. Carmichael, Statistical Methods in Quantum Optics, Vol 1 (Springer, Berlin, 2002).

[3] A. Smirne, H.-P. Breuer, J. Piilo, and B. Vacchini, Phys. Rev. A 82, 062114 (2010).

[4] U. Weiss, Dissipative Quantum Systems (World Scientific, Singapore, 2008).

[5] P. Lambropoulos, G.M. Nikolopoulos, and T.R. Nielsen, Rep. Prog. Phys. 63, 455 (2000).

[6] H.-P. Breuer and B. Vacchini, Phys. Rev. E 79, 041147 (2009); A. Smirne and B. Vacchini, Phys. Rev. A 82, 022110 (2010); A. Kossakowski and R. Rebolledo, Open Syst. Inf. Dyn. 16, 259 (2009).

[7] A.A. Budini, Phys. Rev. A 74, 053815 (2006); H.-P. Breuer, J. Gemmer, and M. Michel, Phys. Rev. E 73, 016139 (2006); H.-P. Breuer, Phys. Rev. A 75, 022103 (2007).

[8] V.P. Belavkin, in Modelling and Control of Systems, edited by A. Blaquière, Lecture Notes in Control and Information Sciences 121 (Springer, Berlin, 1988), pp. 245265; V.P. Belavkin, Phys. Lett. A 140, 355 (1989); V.P. Belavkin, J. Phys. A: Math. Gen. 22, L1109 (1989).

[9] L. Diósi, Phys. Lett. A 129, 419 (1988).

[10] A. Barchielli and V.P. Belavkin, J. Phys. A: Math. Gen. 24, 1495 (1991).

[11] H.J. Carmichael, An Open Systems Approach to Quantum Optics (Springer-Verlag, Berlin, 1993).

[12] K. Mølmer, Y. Castin, J. Dalibard, J. Opt. Soc. Am. B 10, 524 (1993); M.B. Plenio and P.L. Knight, Rev. Mod. Phys. 70, 101 (1998); C. Pellegrini, Stoch. Proc. Appl. 120, 1722 (2010).

[13] C.W. Gardiner and P. Zoller, Quantum Noise: A Handbook of Markovian and Non-Markovian Quantum Stochastic Methods with Applications to Quantum Optics, Springer Series in Synergetics (Springer, Berlin, 2004).

[14] A. Barchielli and M. Gregoratti, Quantum Trajectories and Measurements in Continuous Time: The Diffusive Case, Lecture Notes in Physics 782 (Springer, Berlin, 2009).

[15] H.M. Wiseman and G.J. Milburn, Quantum Measurement and Control (Cambridge University Press, Cambridge, 2010).

[16] H.J. Carmichael, Statistical Methods in Quantum Optics, Vol 2 (Springer, Berlin, 2008).

[17] A. Barchielli, Quantum Opt. 2, 423 (1990); A. Barchielli and A.M. Paganoni, Quantum Semiclass. Opt. 8, 133 (1996); P. Zoller and C.W. Gardiner, in Fluctuations quantiques, edited by S. Reynaud, E. Giacobino, and J. Zinn-Justin, Proceedings of the Les Houches Summer School of Theoretical Physics Session LXIII, 1995 (North-Holland, Amsterdam, 1997), pp. 79-136.

[18] J. Wang, H.M. Wiseman, and G.J. Milburn, Chemical Physics 268, 221 (2001); L. Bouten, R. Van Handel, and M. James, SIAM J. Control Optim. 46, 2199-2241 (2007); H.M. Wiseman and L. Bouten, Quantum Inf. Process. 7, 71-83 (2008).

[19] A.S. Holevo, Statistical Structure of Quantum Theory, Lecture Notes in Physics m 67 (Springer, Berlin, 2001); A. Barchielli, in Open quantum systems III, edited by S. Attal, A. Joye, and C.-A. Pillet, Lecture Notes in Mathematics 1882 (Springer, Berlin, 2006), pp. 207-291.

[20] R. Castro Santis and A. Barchielli, Rep. Math. Phys. 67, 229 (2011).
[21] W.T. Strunz, Phys. Lett. A 224, 25(1996); L. Diósi and W.T. Strunz, Phys. Lett. A 235, 569 (1997); L. Diósi, N. Gisin, and W.T. Strunz, Phys. Rev. A 58, 1699 1998; P. Gaspard and M. Nagaoka, J. Chem. Phys. 111, 5676 (1999); W.T. Strunz, L. Diósi, and N. Gisin, Phys. Rev. Lett. 82, 1801 (1999); A.A. Budini, Phys. Rev. A 63, 012106 (2000); A. Bassi and G.C. Ghirardi, Phys. Rev. A 65, 042114 (2002); J.M. Gambetta and H.M. Wiseman, Phys. Rev. A 66, 012108 (2002); J.M. Gambetta and H.M. Wiseman, Phys. Rev. A 66, 052105 (2002); J.M. Gambetta and H.M. Wiseman, J. Opt. B: Quantum Semiclassical Opt. 6, S821 (2004); W.T. Strunz and T. Yu, Phys. Rev. A 69, 052115 (2004).

[22] L. Diósi, N. Gisin, and W.T. Strunz, Phys. Rev. A 58, 1699 (1998); T. Yu, L. Diósi, N. Gisin, and W.T. Strunz, Phys. Rev. A 60, 91 (1999).

[23] J. Piilo, S. Maniscalco, A. Messina, and F. Petruccione, Phys. Rev. E 71, 056701 (2005); A.A. Budini, J. Phys. B: At. Mol. Opt. Phys. 40, 2671 (2007); J. Piilo, S. Maniscalco, K. Härkönen, and K.-A. Suominen, Phys. Rev. Lett. 100, 180402 (2008); H.-P. Breuer and J. Piilo, EPL 85, 50004 (2009).

[24] J. Piilo, K. Härkönen, S. Maniscalco, and K.-A. Suominen, Phys. Rev. A 79, 062112 (2009); K. Härkönen, J. Phys. A: Math. Theor. 43, 065302 (2010).

[25] J.M. Gambetta and H.M. Wiseman, Phys. Rev. A 68, 062104 (2003); H.M. Wiseman and J.M. Gambetta, Phys. Rev. Lett. 101, 140401 (2008).

[26] L. Diósi, Phys. Rev. Lett. 100, 080401 (2008).

[27] M. Moodley and F. Petruccione, Phys. Rev. A 79, 042103 (2009).

[28] A. Barchielli and C. Pellegrini, J. Math. Phys. 51, 112104 (2010).

[29] A. Barchielli, P. Di Tella, C. Pellegrini, and F. Petruccione, in Quantum Probability and Related Topics, edited by R. Rebolledo, M. Orszag, QP-PQ: Quantum Probability and White Noise Analysis Vol. 27, (World Scientific, Singapore, 2011), pp. 52-67.

[30] A. Barchielli and A.S. Holevo, Stoch. Proc. Appl. 58, 293 (1995).

[31] A. Barchielli, C. Pellegrini, and F. Petruccione, EPL 91, 24001 (2010).

[32] A. Barchielli and M. Gregoratti, Philos. Trans. R. Soc., A 370, 5364 (2012).

[33] D.J. Daley and D. Vere-Jones, An Introduction to the Theory of Point Processes, Volume I: Elementary Theory and Methods (Springer, Berlin, 2003).

[34] A.S. Holevo, Probab. Theory Relat. Fields 104, 483 (1996); C.M. Mora and R. Rebolledo, Ann. Appl. Prob. 18, 591 (2008); F. Fagnola and C.M. Mora, Stochastic Schrödinger equations and applications to Ehrenfest type theorems, arXiv:1207.2939v1 [quant-ph].

[35] J.T. Stockburger and C.H. Mak, J. Chem. Phys. 110, 4983 (1998); A.A. Budini, Phys. Rev. E 72, 056106 (2005).

[36] H.J. Kimble and L. Mandel, Phys. Rev. A 15, 689 (1977).

[37] R.M. Howard, Principles of Random Signal Analysis and Low Noise Design, The Power Spectral Density and its Applications (Wiley, New York, 2002)

[38] A. Barchielli and G. Lupieri, J. Math. Phys. 41, 7181 (2000). 